\title{
The Golden Age of Cataclysmic Variables and Related Objects (some critical comments)
}

\section{Franco Giovannelli*}

INAF - Istituto di Astrofisica e Planetologia Spaziali, Via del Fosso del Cavaliere, 100, 00133

Roma, Italy

E-mail: Eranco.giovannellidinaf.it

In the last few years we assisted to a substantial improvement on the number of cataclysmic variables (CVs) discovered, but only few exciting results have been obtained such as the discovery of a white dwarf pulsar in AR Sco. In this paper I will comment, from a personal point of view, some news about the route to be followed in the investigations on CVs and Related Objects.

The paper will be developed as follows:

- Introduction

- The use of wisdom in Physics

- Some comments on Cataclysmic Variables

- Classical and Recurrent Novae

- Progenitors of Type-Ia Supernovae

- Nuclear Reactions in Stars

- Some Open Questions

- Conclusions

The Golden Age of Cataclysmic Variables and Related Objects V (GOLDEN2019)

2-7 September 2019

Palermo, Italy

\footnotetext{
* Speaker.
} 


\section{Introduction}

The first variable star was described thousands of years ago.

The oldest known record of a variable star dates back about 3,200 years. This record of a now-famous variable star, the eclipsing binary star Algol in the constellation Perseus, is contained in an ancient calendar that lists auspicious and inauspicious days, or as we would view them today, lucky and unlucky days.

Johannes Phocylides Holwarda (February 19, 1618 - January 22, 1651) was a Frisian astronomer, physician, and philosopher (Fig. 1). He was a professor of philosophy at the University of Franeker from 1639 to 1651.

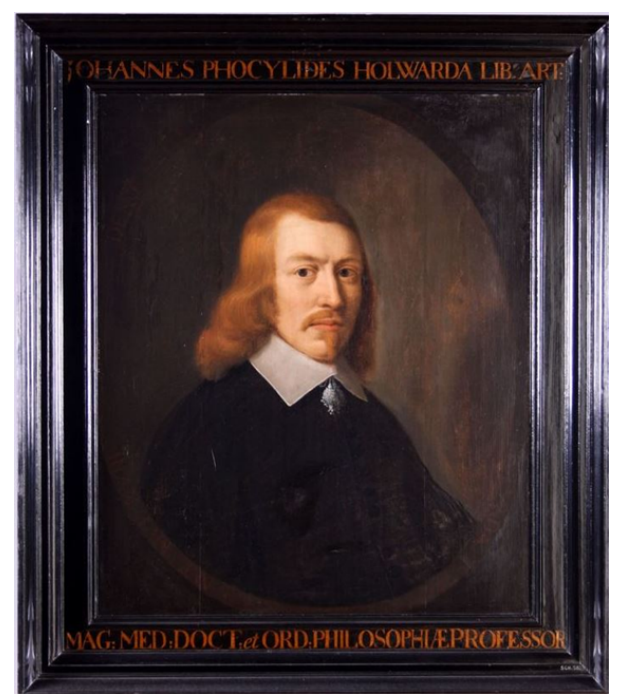

Figure 1: Johannes Phocylides Holwarda.

Born in Holwerd, he is best remembered for his discovery of the length of Mira's (Omicron Ceti's) varying appearance cycle. In a systematic study in 1638, he found that Mira disappeared and reappeared in a varying cycle of about 330 days.

All the stars are practically variables with different level of variations in luminosity and in time. It is possible to distinguish variable stars in two categories:

- Intrinsic variable stars in which the mechanisms that cause luminosity variations involve the physical properties of the stars.

- Extrinsic variable stars in which the mechanisms that cause luminosity variations involve extrinsic factors such as a companion star that eclipses a "normally relatively stable star" periodically.

Figure 2 shows an interesting classification of variable stars in Group, Class, and Type (Christoforou, 2017)).

This is a convenient classification that however masks the real continuity among all the variable stars. Indeed, in nature everything is linked with different strength of the connections. In fact there is a Bridge between the Big Bang and Biology. Indeed, independent of the origin of our Universe, 


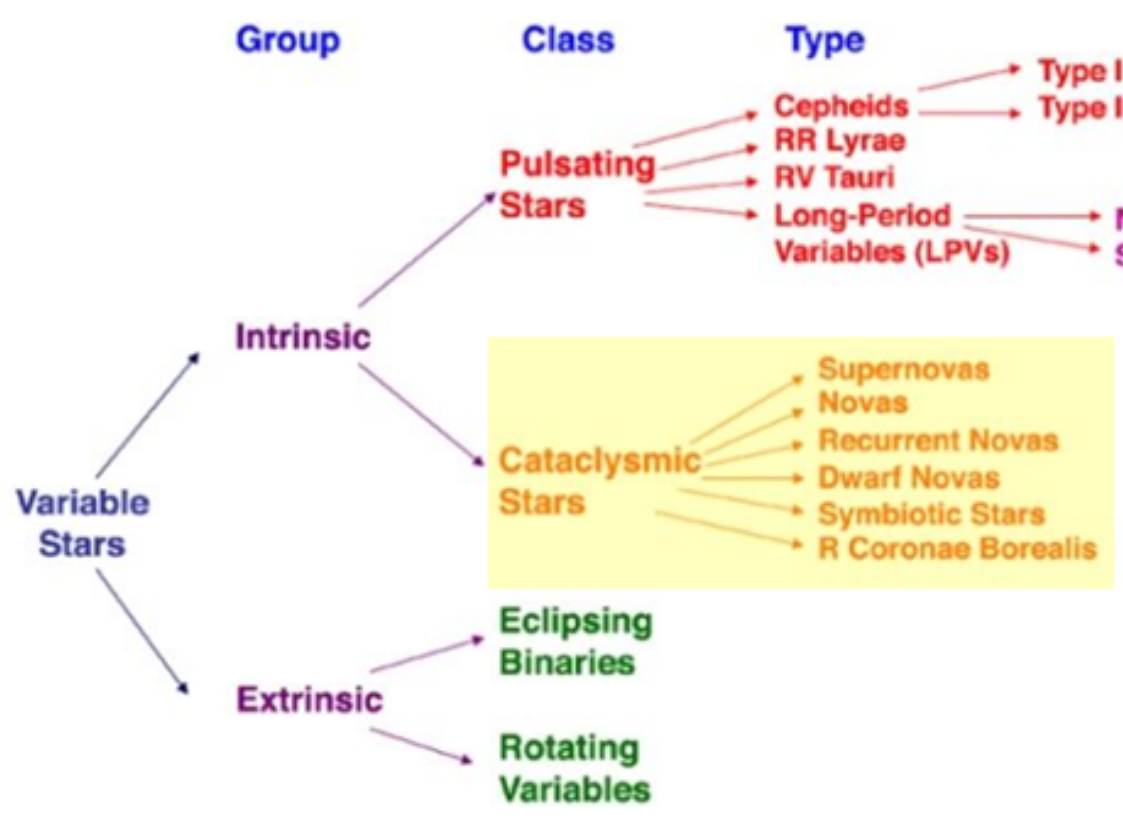

Figure 2: Variable stars: Group, Class, and Type (adapted from Christoforou, 2017). Light yellow rectangle marks the cataclysmic stars.

we are here in this planet. This is the demonstration that the bridge really exists (Giovannelli, 2001).

Therefore two fundamental questions arise: i) How to cross this Bridge? ii) What are the experimental tools for understanding the pillars of this Bridge?

In order to cross this bridge, as always when we cross a bridge, we MUST advance slowly, step by step, with continuity, because everything is smoothly linked in the "magma" of the Universe, from the infinitely small to infinitely big, as sketched in Fig. 1 (adopted from Giovannelli \& SabauGraziati, 2017a after Rees, 1988).

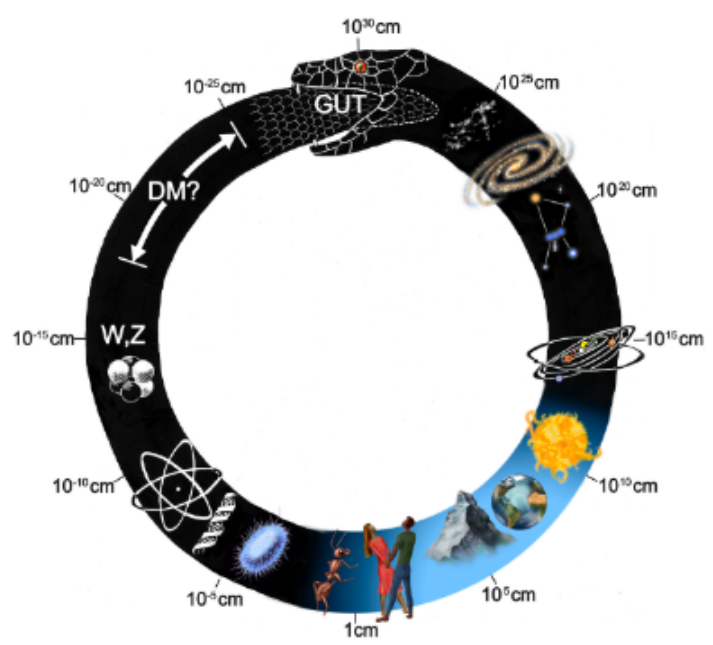

Figure 3: From the infinitely small to infinitely big (adopted from Giovannelli \& Sabau-Graziati, 2017a after Rees, 1988). 

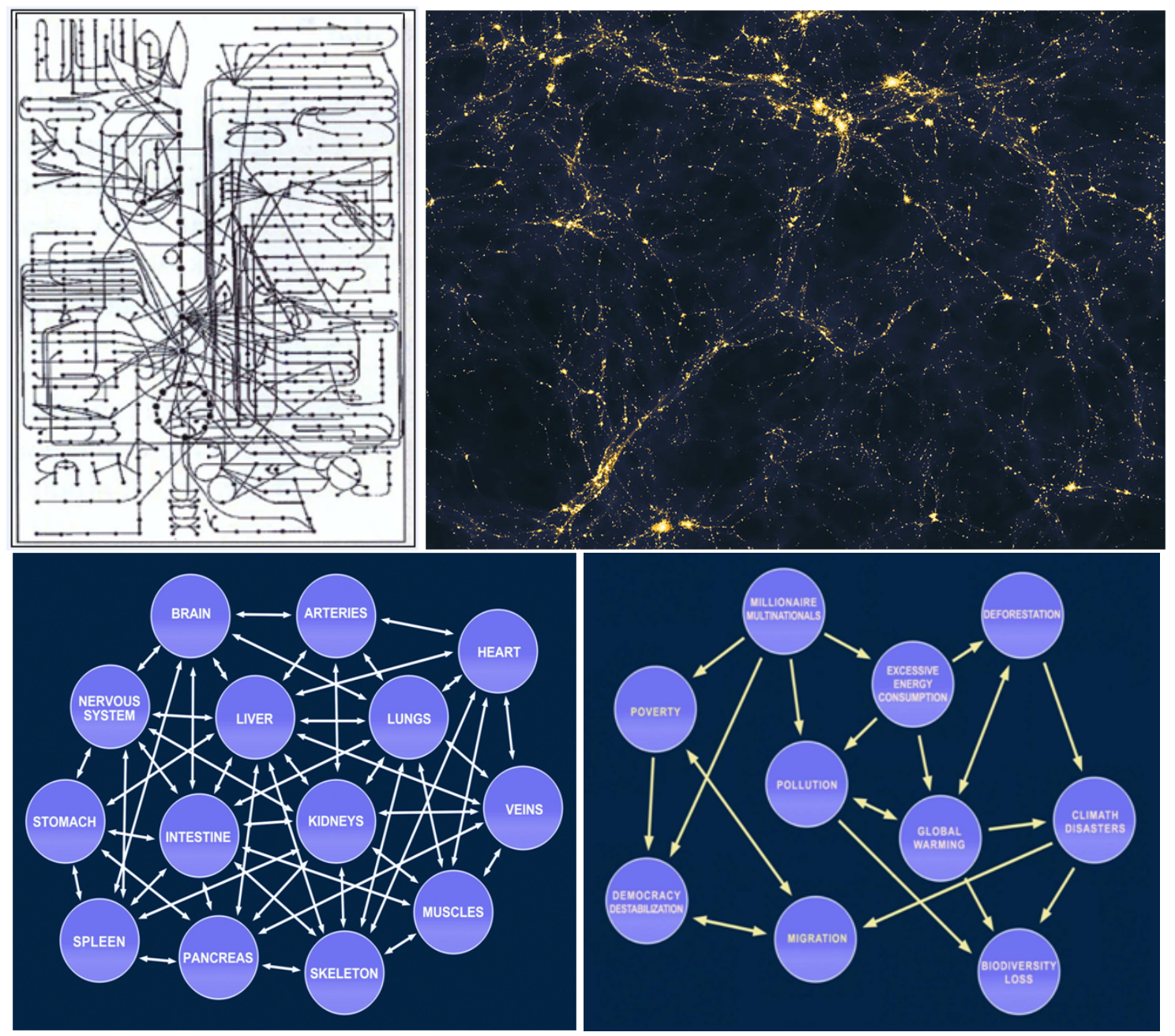

Figure 4: Upper left panel: Section of the metabolic network of a "simple" bacterium (Luisi \& Capra, 2014). Upper right panel: the "cosmic network" [Credit: Andrew Pontzen and Fabio Governato, 2014; https://www.flickr.com/photos/uclmaps/15051460475/]. Lower left panel: the human body network. Lower right panel: the human society network (Luisi \& Capra, 2014).

In nature, nothing is isolated. Everything is related to the surrounding environment in a more or less strong way. However, the link exists. Fig 4 (upper left) shows a section of the metabolic network of a "simple" bacterium. Note that each point (each chemical compound) is connected to any other point through the complexity of the network (Luisi \& Capra, 2014). Fig. 4 (Upper right) shows the cosmic network: each point is connected to any other point through the complexity of the network (Credit: Andrew Pontzen and Fabio Governato, 2014; https://www.flickr.com/photos/uclmaps/15051460475/). The large-scale structure of the Universe, as traced by the distribution of galaxies, is now being revealed by large-volume cosmological surveys. The structure is characterized by galaxies distributed along filaments, the filaments connecting in turn to form a percolating network. This image is a computer simulation of an area of space more than 50 million light-years across, presenting a possible large-scale distribution of light sources in the universe. Precise relative contributions of galaxies and quasars are unclear. The objective of Shandarin, Habib \& Heitmann (2010) was to quantitatively specify the underly- 
ing mechanisms that drive the formation of the cosmic network. By combining percolation-based analyzes with N-body simulations of gravitational structure formation, they elucidate how the network has its origin in the properties of the initial density field (nature) and how its contrast is then amplified by the nonlinear mapping induced by the gravitational instability (nurture). ${ }^{(*)}$

\footnotetext{
${ }^{(*)}$ In statistical physics and mathematics, percolation theory describes the behavior of a network when nodes or links are removed. This is a type of phase transition, since at a critical fraction of removal the network breaks into significantly smaller connected clusters. The percolation threshold is a mathematical concept in percolation theory that describes the formation of long-range connectivity in random systems. Below the threshold a giant connected component does not exist; while above it, there exists a giant component of the order of system size. Percolation theory has been used in astrophysics before the study of the large-scale structure in the Universe as a way to characterize voids, walls, and filamentary structure, an analogy explored early in the history of gravitational instability theory (Shandarin,1983) and more recently in the context of modern structure formation theory (e.g. Sahni, Sathyaprakash \& Shandarin, 1997; Shandarin et al., 2006; Shandarin, Habib \& Heitmann, 2010; Falck \& Neyrinck, 2015). In that case, it is useful in describing the distribution of low-density regions, but the filling factor of those regions (the quantity of most interest in percolation processes) is only indirectly observable through its implications for the galaxy distribution. However, it is possible to see that many of the results of these studies are relevant to the percolation of ionized structures during reionization (Furlanetto \& Oh, 2016).
}

Fig. 4 (Lower left) shows the human body network: each point (organ) is connected to any other point (organ) through the complexity of the network (Luisi \& Capra, 2014). Fig. 4 (Lower right) shows the human society network: each point is connected to any other point through the complexity of the network (Luisi \& Capra, 2014).

All the components of the Universe follow the cycle: birth, growth, aging, death like Humans that belong to the Universe. Therefore for a complete understanding of the history of the Universe it is necessary to search along that cycle.

In the long review published by Giovannelli (2017) an exhaustive discussion about the importance of considering CVs as gravimagnetic rotators was reported, as well as the hints for considering all the CVs as a manifestation of physical conditions of binary systems smoothly connected along their evolutionary path, independent of the popular classification in Polar (Ps), Intermediate Polars (IPs) and non-magnetic CVs (NMCVs).

In the following I want to remark some important experimental facts that, if used with some wisdom, can produce interesting results otherwise reached in a difficult way. This imply the use of results coming from different experiments and then used without blinkers.

\section{The use of wisdom in Physics}

A clear example of the use of wisdom in physics is coming from the history of the CV SS Cygni. In spite that it is the most observed CV since the end of the 19th century (Parkhurst \& Zaccheus, 1900; Zuckerman, 1961), there is still a controversy about its nature. An extensive review about SS Cyg was published by Giovannelli \& Martinez-Pais (1991).

Probably, the "mistake" about the nature of SS Cyg born after the publication of a paper in Nature by Bath \& van Paradijs (1983) where SS Cyg was classified as dwarf nova (DN) on the basis 
of optical behaviour, typical of DNe. This paper originated a bandwagon effect in the literature (see Michael Friedjung's comment in the first historical Frascati Workshop 1984: Giovannelli, 1985) that "obliged" almost all the subsequent authors to start the papers saying that SS Cyg is a DN (NMCVs), without paying attention to other possibilities well documented in the so-called second class literature.

Therefore, my suggestion is to reconsider the problem about the nature of SS Cyg without any a priori bias.

A recent paper by Hill et al. (2017) suggests to me to tell the history of the determination of the parameters of SS Cygni, and more, especially for the benefit of younger colleagues. Indeed, Fig. 5 shows the system parameters of SS Cyg, and in the last line, marked with a light red rectangle, the parameters derived by Giovannelli et al. (1983) are reported. These parameters, in spite of their origin from several measurements coming from different wavelength regions, are still valid if compared to "more accurate" experimental sources.

Table 2. System parameters of SS Cyg, with columns 1-7 listing the authors, the systemic velocity, the inclination, the radial-velocity semi-amplitude of the secondary star, the primary mass, the secondary mass and the mass ratio.

\begin{tabular}{lcccccc}
\hline \multicolumn{1}{c}{ Author } & $\gamma\left(\mathrm{km} \mathrm{s}^{-1}\right)$ & $i$ (degrees) & $K_{2}$ & $M_{1}\left(\mathrm{M}_{\odot}\right)$ & $M_{2}\left(\mathrm{M}_{\odot}\right)$ & $q=\frac{M_{2}}{\mathbb{Y}_{1}}$ \\
\hline This work & -15.2 & 45 & 163.9 & 0.94 & 0.59 & 0.628 \\
Hessman et al. (1984) & $-15.1 \pm 1$ & - & $155 \pm 2$ & - & - & 0.595 \\
North et al. (2002) & $-13.09 \pm 2.88$ & - & $165 \pm 1$ & - & - & $0.68 \pm 0.02$ \\
Bitner et al. (2007) & $-13.1 \pm 2.9$ & $45-56$ & $162.5 \pm 1$ & $0.81 \pm 0.19$ & $0.55 \pm 0.13$ & $0.685 \pm 0.015$ \\
Martinez-Pais et al. (1994) & - & - & $162.5 \pm 3$ & - & - & - \\
Giovannelli et al. (1983) & - & $40_{-2}^{+1}$ & - & $0.97_{-0.05}^{+0.14}$ & $0.56_{-0.03}^{+0.05}$ & $0.58_{-0.10}^{+0.12}$
\end{tabular}

Figure 5: System parameters of SS Cyg (adapted from Hill et al., 2017).

As discussed by Smak (1985), emission lines are the most prominent features in the optical and ultraviolet spectra of CVs. Typically these are lines of $\mathrm{H}$, neutral and ionized $\mathrm{He}$, and ionized Ca. Their intensities are different in different systems. In the case of CVs with accretion disk around the white dwarf, the emission lines are often double peaked.

Indeed, the behaviour of the emission lines demonstrates that the transferred material does in fact produce a true disk rather than an amorphous cloud. Smak (1969) and Huang (1972) calculated the emission line profiles expected from gas disks of CVs and found that they should be double peaked and should have extensive wings.

The double component comes from the disk and originates from the surface of a flat, Keplerian disk (Smak, 1981), like shows in Fig. 6. Almost regardless of the radial distribution of the emitting atoms, the half-separation of the two peaks gives, to within $\sim 15 \%$ (Smak, 1969; Huang, 1972), the rotational velocity of the outer edge of the disk, $V_{d} \sin i$ (where $i$ is the orbital inclination). For $\mathrm{V}_{\mathrm{d}}$ we have:

$$
V_{\mathrm{d}}^{2}=\frac{G M_{\mathrm{WD}}}{R_{\mathrm{d}}}
$$

and for the observed velocity $\mathrm{V}_{\mathrm{obs}}$, we have: 


$$
\begin{aligned}
& \frac{1}{2} \frac{\Delta \lambda}{\lambda}=\frac{V_{\mathrm{obs}}}{c} \\
& V_{\mathrm{obs}}=V_{\mathrm{d}} \sin i
\end{aligned}
$$

where:

$\mathrm{R}_{\mathrm{d}}=$ outer radius of the disk; $\Delta \lambda=$ separation of the peaks; $\mathrm{c}=$ light velocity; $\mathrm{V}_{\mathrm{obs}}=$ observed velocity; $\mathrm{G}=$ gravitational constant; $\mathrm{M}_{\mathrm{WD}}=$ mass of the white dwarf; $i=$ orbital inclination angle.

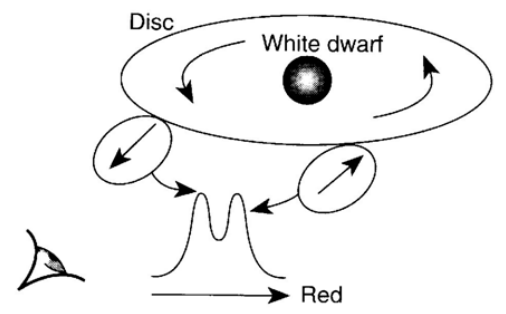

Figure 6: Sketch of the production of doubling in the emission lines from a flat, Keplerian accretion disk around the white dwarf in NMCVs (Smak, 1981, 1985).

Giovannelli et al. (1983), with an hazardous operation for that time, started to collect all information coming from different wavelength regions for determining the parameters of SS Cyg, namely:

- their $\mathrm{V}_{\mathrm{obs}}=192 \pm 10 \mathrm{~km} \mathrm{~s}^{-1}$ from the doubling observed in the Balmer emission lines in spectra taken during a quiescent phase of the system, as supported by the AAVSO measurements.

- The optical pulsations measured during different states of SS Cygni and ranging from $7.3 \mathrm{~s}$ (Hildebrand, Spillar \& Stiening, 1981) to 10.9 s (Horne \& Gomer, 1980; Patterson, 1979, 1981; Giovannelli, 1981).

- The X-ray pulsation of $9.735 \pm 0.002 \mathrm{~s}$ measured by Patterson, Robinson \& Kiplinger (1978)

- The available orbital data and the ratio between the external radius of the disk $\mathrm{R}_{\mathrm{d}}$ and the separation, a, between the two components of the system, determined by Stover et al. (1980), Kiplinger (1979a,b), and Joy (1956).

- The mass-radius relationship for the white dwarf by Hartle \& Thorne (1968).

- The mass-radius relationship for the secondary star by Plavec (1968).

They, by using Hartle \& Thorne (1968) mass-radius relationship for the white dwarf and the minimum and maximum values of the optical pulsations, they limited the values of the orbital 

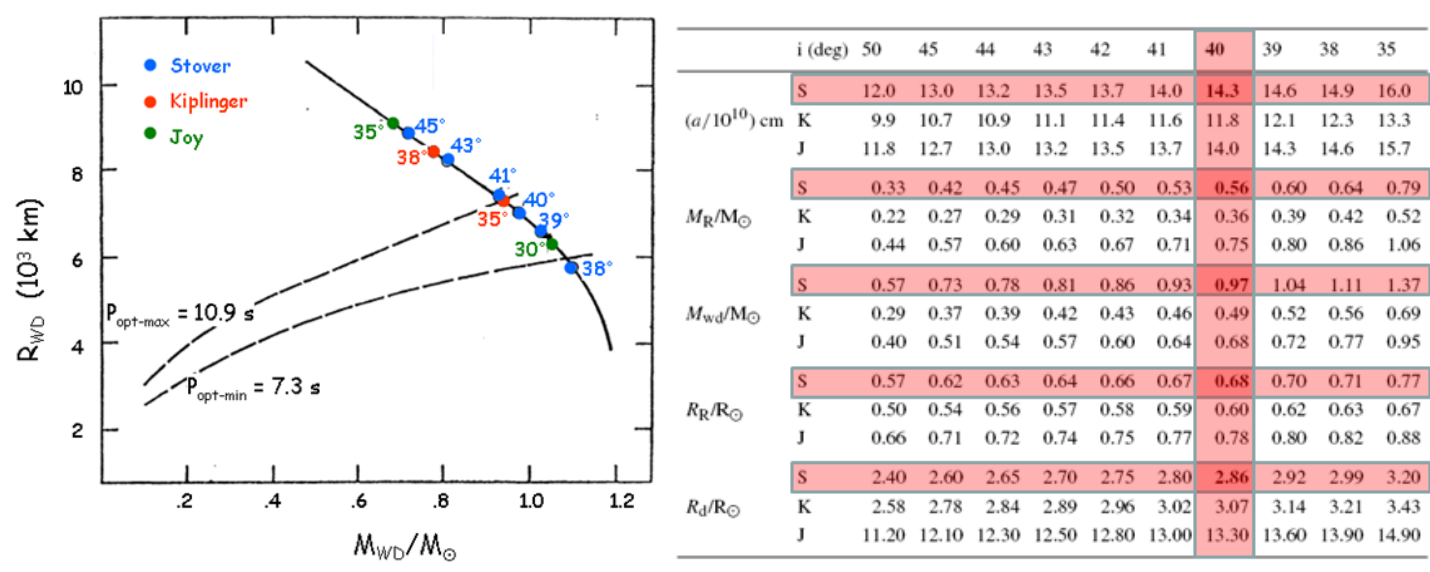

Figure 7: Left panel: mass-radius relationship for the WD and possible orbital inclination angles delimited by the values of optical pulsations (Adapted from Giovannelli et al., 1983). Right panel: table with orbital parameters for different values of orbital inclination angles. Light-red horizontal rectangles show the most probable values, coming from Stover et al. (1980), and the light-red vertical rectangle shows the orbital parameters of SS Cyg (adapted from Giovannelli et al., 1983).

inclination angle between $38^{\circ}$ and $41^{\circ}$ (see Fig. 7, left panel). Then, if the X-ray pulsation of 9.7 s gives an upper limit to the Keplerian period at dwarf nova surface (Cordova et al., 1980), the lower limit of the mass of the white dwarf is $0.9 \mathrm{M}_{\odot}$. Thus, they constructed a table in which are reported all the parameters for different values of the orbital inclination angle (Fig. 7, right panel). Therefore, the orbital parameters of SS Cyg can be derived from the column marked with a light-red rectangle.

Thus, the orbital parameters of SS Cyg are:

$\mathrm{i}=40_{-2^{\circ}}^{\circ} ; \mathrm{a}=14.3_{-0.3}^{+0.6} \times 10^{10} \mathrm{~cm} ; \mathrm{M}_{\mathrm{R}}=0.56_{-0.03}^{+0.08} \mathrm{M}_{\odot} ; \mathrm{M}_{\mathrm{WD}}=0.97_{-0.05}^{+0.14} \mathrm{M}_{\odot} ; \mathrm{R}_{\mathrm{R}}=0.68_{-0.01}^{+0.03} \mathrm{R}_{\odot}$; $\mathrm{R}_{\mathrm{d}}=2.86_{-0.06}^{+0.13} \times 10^{10} \mathrm{~cm}$.

From the $\mathrm{H}_{\beta}$ in emission, Giovannelli et al. (1983) determined the width at the level of continuum as $\approx 2500 \mathrm{~km} \mathrm{~s}^{-1}$. This imply a value of the innermost part of the accretion disk of $\mathrm{R}_{\mathrm{in}}$ $\approx 3.6 \times 10^{9} \mathrm{~cm}$. This value is consistent with the radius of the WD $\left(\mathrm{R}_{\mathrm{WD}}=5 \times 10^{8} \mathrm{~cm}\right)$ derived by Martinez-Pais et al. (1994). Bisikalo et al. (2008) by using Doppler tomography in SS Cyg in quiescence found $R_{\text {in }}=(2.6-3.3) \times 10^{9} \mathrm{~cm}$, in agreement with the value derived, with less elaborate mode, by Giovannelli et al. (1983).

From the goodness of Giovannelli et al. (1983) parameters several other results are coming out, like the position of SS Cyg in the plot of the Keplerian period at the surface of the WD, contrary to the position plotted by Patterson (1981), who used the Chandrasekhar limit for the WD mass of SS Cyg (see Fig. 8, left panel). Another interesting results is reported in Fig. 8 (right panel). Indeed, if we use the diagram $\dot{\mathrm{M}}_{\mathrm{tr}}$ vs $\mathrm{R}_{\mathrm{d}}$ (the mean mass-transfer rate vs. radius of the accretion disk) and the critical accretion rate marked with red line in the right panel of Fig. 8 (Schreiber \& Lasota, 2007):

$\dot{\mathrm{M}}_{\text {crit }}=9.5 \times 10^{15} \mathrm{~g} \mathrm{~s}^{-1} \times \mathrm{R}_{10}^{2.68} \times \mathrm{M}_{\mathrm{WD}}\left(\mathrm{M}_{\odot}\right)$

(being $\mathrm{R}_{10}$ the accretion disk radius in unit of $10^{10} \mathrm{~cm}$ ),

and the critical accretion rate for SS Cyg derived with the Giovannelli et al. (1983) parameters is: $\dot{\mathrm{M}}_{\text {crit }}(\mathrm{SS} \mathrm{Cyg})=1.6 \times 10^{17} \mathrm{~g} \mathrm{~s}^{-1}$ 
Thus, the position of SS Cyg is marked with the light-red rectangle, according to experimental error bars, in the right panel of Fig. 8.
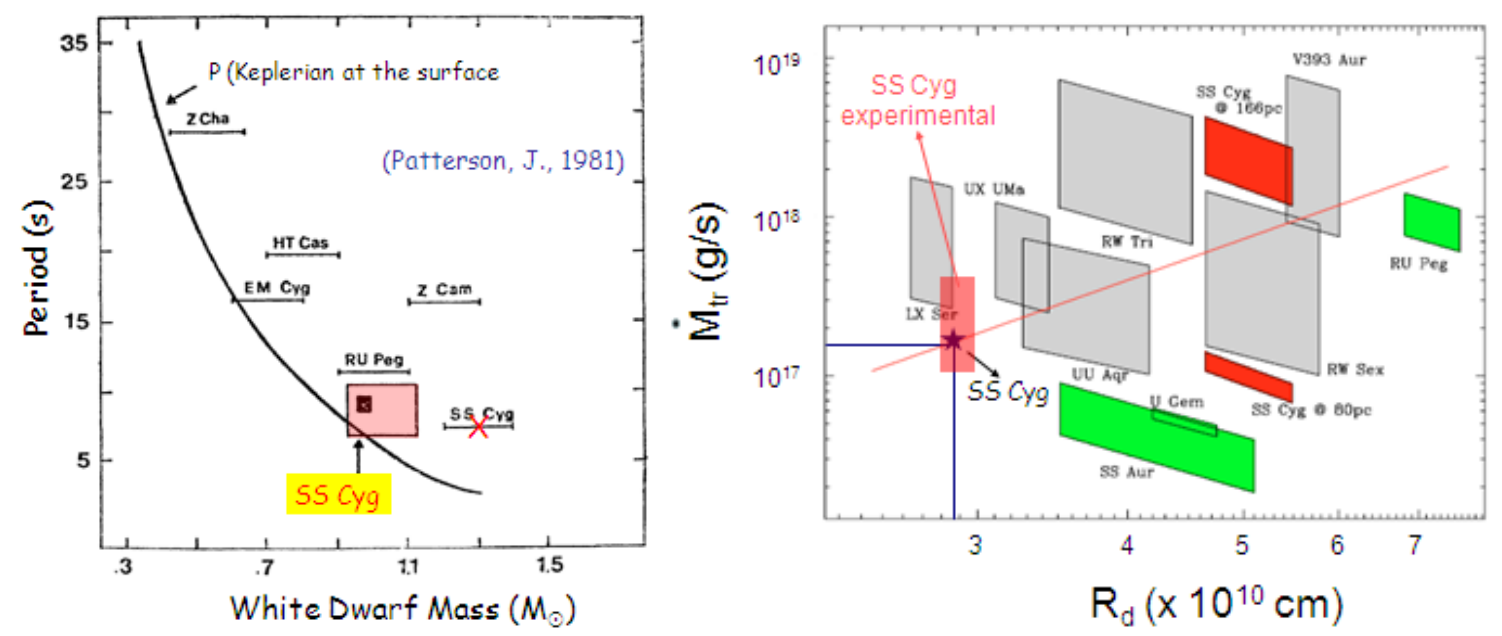

Figure 8: Left panel: Keplerian period at the WD surface versus WD mass (Giovannelli et al., 1983 after Patterson, 1981). Right panel: (adapted from Schreiber \& Lasota, 2007).

In my opinion, another open question is that about the nature of SS Cyg: NMCV or MCV (IP). There are many papers that discuss such a problem, like those by Giovannelli $(1985,1996)$, Lombardi, Giovannelli \& Gaudenzi (1987), Giovannelli, Martinez-Pais \& Sabau-Graziati (1992), Gaudenzi et al. (2002), Giovannelli \& Sabau-Graziati (1999, 2012a,b). In particular in the latter two papers (2012a,b) a deep discussion of the pro and contra about the IP nature of SS Cyg is reported.

However, since I would like to convince a reader that it is always appropriate to use wisdom in every event of life and in particular in science, I ask a question below to which each one will be able to answer as best he can, however trying to avoid prejudices.

INTEGRAL observatory measured hard X-ray emission from $23 \mathrm{MCVs}$, with the exception of SS Cyg, classified as NMCV, as shown in Fig. 9 (Scaringi et al., 2010). Thus, my question is: why all the CVs measured by the INTEGRAL are MCVs, but SS Cyg? And, if SS Cyg is NMCV, why INTEGRAL did not measure any other NMCV?

\section{Some comments on CVs}

One of the most exciting problem about the compact objects, such as WDs and NSs, is connected with the rotation and spin-down rates. Soft Gamma Ray Repeaters (SGRs) and Anomalous X-ray Pulsars (AXPs), deeply discussed in the review paper by Giovannelli \& Sabau-Graziati (2019), have been considered either as fast rotating WDs or NSs.

Briefly, SGRs and AXPs are a class of compact objects that show interesting observational properties (e.g. Mereghetti, 2008; Giovannelli \& Sabau-Graziati, 2019), namely: rotational periods, $\mathrm{P}$, in the range 5.5-11.8 s - contrary to the larger spread of those of HMXBs $(0.069$-few $\times 10^{3} \mathrm{~s}-$ and spin-down rates $\dot{\mathrm{P}}$ in the range $(0.05-10) \times 10^{-11} \mathrm{~s} \mathrm{~s}^{-1}$, larger than those of 'ordinary pulsars' $\left(\dot{\mathrm{P}} \sim 10^{-15} \mathrm{~s} \mathrm{~s}^{-1}\right)$; strong outbursts of energies in the range $\sim 10^{41}-10^{43} \mathrm{erg}$, and 


\begin{tabular}{|c|c|c|c|c|c|c|c|c|c|c|c|}
\hline Nona" & $\begin{array}{c}a, \beta^{8} \\
\text { ants posities) }\end{array}$ & $6 \mathrm{w}$ & $\frac{e^{2} e^{2}}{0}$ & Map oble & 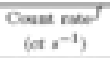 & $\begin{array}{c}\text { Expoute } \\
\text { (bs) }\end{array}$ & $\sum_{x \rightarrow \infty}^{n=0}$ & ${ }_{(\min )}^{n+4}$ & $F_{(0)}$ & $\begin{array}{c}\text { Dintand } \\
\text { (yx) }\end{array}$ & Rets \\
\hline 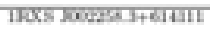 & 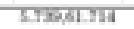 & IF & I & एकरम & वाडसका & $\mathrm{ISE}$ & क्या & आक्र & प्राज & Iा6 & 15214 \\
\hline v70 Cas & 7200,5000 & iv & as & Bธนแ3 & $160=601$ & 300 & 5sis & 3004 & $\sin \pi$ & $=6$ & 1.29 \\
\hline XYAnt & Hovr.13.45T & tp & 11 & Eciss) & $0.53=0.12$ & 119 & $2 \mathrm{NS}$ & seaker & 30620 & 616 & 1.26 \\
\hline GK NE & $32 \pi .4395$ & W/DN & 17 & E:4.7) & $0 x \pm 60$ & $2 \pi$ & 14 & 20es 4 & $x_{134}$ & - & [1.:] \\
\hline TV Cal. & k2.25:-32.215 & IP & a. & В4116 & $0.6 \pm 0.08$ & $2 w$ & ox $x$ & 250.184 & เต1 & $\mathbf{m e}$ & H...7. \\
\hline $\mathrm{Tw} P \mathrm{Pk}$. & Kanec.57908 & IPINY" SAl:. & 25 & aids? & $0.3 \pm 0.67$ & 30 & 1.61 & 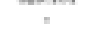 & 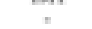 & 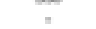 & {$[1,22$, , 11$]$} \\
\hline Bर $\mathrm{Cam}$ & 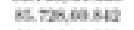 & $A D$ & 12 & Es(a.1) & $0 \omega=0.11$ & 160 & 27 & $2 n=0$ & Itses.4 & 160 & [1.] ] \\
\hline gu Can & $96.316 .73 \times 67$ & $\mathbb{w}$ & 0.6 & Best & $0.24=000$ & sto & 120 & 203104 & 1587.34 & 40 & 1.211 \\
\hline SWIFT 30 mos - 1821 . & 113.12.215:13 & iv & 16 & Bsicen & $0 \times 0=000$ & $\$ 0$ & 200 & $x \leq 04$ & 51142 & 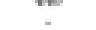 & 1.211 \\
\hline vout Ces & mase- $\leq 5>0$ & p & as & Bis.4) & $a x+600$ & jes & 9.46 & sutsira & ตอง 697 & so & {$[17$} \\
\hline $\tan 114036-\operatorname{sen} 2$ & men,ssm & p & 20 & 14 (a19) & $0.27=000$ & sess & is & 170.36 & 18061.6 & 10 & 1.214 \\
\hline xylap & $292003-6.451$ & iv & as & nesis & 172000 & sat & 48 & 51.84 & $\cos 01$ & 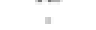 & 1.215 \\
\hline vako opd & 258.172 .24200 & ip & 21 & Baำ. & $0.65 \pm 002$ & 45s & 165 & 204.45 & 2076 & 100 & 1.216 \\
\hline 谓 $17 x-\alpha s$ & 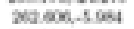 & w & at & $\mathrm{B}(\mathrm{n}) \mathrm{s}$ & $0.55 \pm 606$ & 140 & 4.46 & $\cos \pi$ & $m$ & - & 1.217 \\
\hline vav? Ond & $\times a 206-19244$ & D/Ning & 23 & kato.1) & $0.25+609$ & sea & $a s e$ & $=$ & . & $=$ & 1.218 \\
\hline 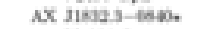 & 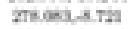 & $?$ & 11 & ats.s? & $0.00=000$ & 300 & $a x$ & 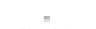 & . & 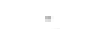 & $0,2,19,34$ \\
\hline vizm Svy & $209713-31.15$ & iv & as & ness? & $1.45=600$ & 2205 & $7 \pi$ & messes & 246 & 190 & [1. \\
\hline Vian Ad & $256.062,-10.21$ & $A D$ & a.s & $\mathrm{Bs}(\mathrm{x}) \mathrm{s}$ & $0 \omega=0.07$ & $8 \infty$ & 37 & nises & Easo. & 200 & $0,2,21,2 \pi$ \\
\hline vawe ces & 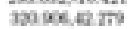 & E. & 1 & siosat & $021 \pm 600$ & $1 \in 6$ & 1.13 & 448.804 & 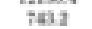 & . & 1.2.2nad \\
\hline 12Xs manuititsoegs & maksim & IP & 0.3 & Resses) & 0 estem & tase & 169 & a1 ua & $\sin x_{2}$ & . & 1.235 \\
\hline ss cye & $\cos \cos x \sec$ & is & 0.6 & Bszon & 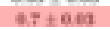 & 1eed & 176 & 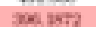 & . & 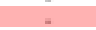 & $1.2 \times$ \\
\hline Fo $\mathrm{Aq}_{4}$ & sustustat & IP & 1.7 & awo.1) & 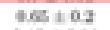 & 54 & 145 & 200506 & 12045 & 200 & 1.227 \\
\hline AO P. & $38 x+5,-194$ & iv & 13 & Bets? & $0.43 \pm 0.11$ & 100 & 231 & 2ns $x=1$ & $\operatorname{sos}_{3}$ & 20 & $1.2 \times 2 x$ \\
\hline
\end{tabular}

Figure 9: List of CVs measured by the INTEGRAL observatory in the hard X-ray range (adapted by Scaringi et al. (2010).

giant flares in SGRs of energies in the range $\sim 10^{44}-10^{47} \mathrm{erg}$, not observed in 'ordinary pulsars'. Spin periods of AXPs are monotonically increasing on timescales of $\sim 10^{4}-4 \times 10^{5} \mathrm{yr}$.

Measurements of the spin down rates of SGRs and AXPs have been interpreted as evidence of very strong magnetic fields at the collapsed object poles, roughly two orders of magnitude greater than those of the 'normal' X-ray pulsars. For this reason they are now known as 'magnetars'. Their derived magnetic field intensity is $\sim 10^{14}-10^{15} \mathrm{G}$.

But with the observation of SGR 0418+5729 (Rea et al., 2010) the situation about the nature of magnetars became controversial. Indeed, SGR 0418+5729 shows a rotational period of $9.08 \mathrm{~s}$, an upper limit of the first time derivative of the rotational period $\dot{\mathrm{P}}<6.0 \times 10^{-15} \mathrm{~s} \mathrm{~s}^{-1}$, and X-ray luminosity $\mathrm{L}_{\mathrm{X}}=6.2 \times 10^{31} \mathrm{erg} \mathrm{s}^{-1}$. Thus, with the magnetar model based on a NS of fiducial parameters $\mathrm{M}=1.4 \mathrm{M}_{\odot}, \mathrm{R}=10 \mathrm{~km}$, and a moment of inertia $\mathrm{I}=10^{45} \mathrm{~g} \mathrm{~cm}^{2}$ it is necessary a magnetic field intensity larger than the critical field for vacuum polarization $B_{\text {crit }}=m_{e}^{2} c^{3} /(e \hbar)=$ $4.4 \times 10^{13} \mathrm{G}$ in order to explain the observed X-ray luminosity in terms of the release of magnetic energy (Duncan \& Thompson, 1992; Thompson \& Duncan, 1995). Therefore, the upper limit of the surface magnetic field of SGR $0418+5729$ is $B<7.5 \times 10^{12} \mathrm{G}$ if it is a NS, well below the critical field in the magnetic scenario.

Therefore, starting from the pioneering works of Morini et al. (1988) and Paczynski (1990) about the description of 1E 2259+586 as a WD, Malheiro, Rueda \& Ruffini (2012) discussed this interpretation in the case of SGR $0418+5729$ by using the measurements obtained by Rea et al. (2010), and they demonstrated that this source can be an authentic 'Rosetta Stone', a powerful discriminant for alternative models of SGRs and AXPs. Such an interpretation could be extended to all SGRs and AXPs . Fiducial parameters of these WDs were assumed as $\mathrm{M}=1.4 \mathrm{M}_{\odot}, \mathrm{R}=10^{3}$ $\mathrm{km}$, moment of inertia $\mathrm{I}=10^{49} \mathrm{~g} \mathrm{~cm}^{2}$, and magnetic fields $\mathrm{B} \gtrsim 10^{7} \mathrm{G}$, inferred from the observed rotational periods and spin-down-rates. Later, Rueda et al. (2013) discussed the problem of a WD merger as progenitor of the anomalous X-ray pulsar 4U 0142+61. Boshkayev et al. (2013) discussed "On general relativistic uniformly rotating White Dwarfs".

This problem about the rotating WDs and NSs in General Relativity have been deeply discussed in the PhD thesis of Boshkayev (2013), and the references therein. 
Rea et al. $(2012,2013,2014)$ reported measurements from SGR 1822-1606, again from SGR 0418-5729, and 3XMM J185246.6+003317. These, are the three lowest magnetic field magnetars: $\mathrm{B} \simeq 2.7 \times 10^{13} \mathrm{G}, \mathrm{B} \simeq 6 \times 10^{12} \mathrm{G}$, and $\mathrm{B}<4.1 \times 10^{13} \mathrm{G}$, respectively. These low magnetic field magnetars could be considered as a link between the 'normal' pulsars and 'magnetars' following the main idea I discussed in the introduction: each cosmic source is linked with continuity among the others classified, for convenience, as belong to different classes.

The latter authors discussed their data under the hypothesis that the compact object of the SGRs is a neutron star, avoiding to consider the possibility that could be a white dwarf, like discussed by Boshkayev, Rueda \& Ruffini (2015) in the case of SGR 0418+5729.

The open questions about magnetars are numerous, namely: i) What are the distances of the Galactic magnetars? Then what is the Energetics? ii) What is the number-intensity relation for giant magnetar flares? iii) What are the SGR and AXP birth rate? What are their lifetimes? How many SGRs and AXPs are in the Milky Way? iv) What kind of supernova produces a SGR or an AXP? v) What is the relation between SGRs and AXPs? Does one evolve into the other, or are they separate manifestations of magnetars? vi) Are really the collapsed objects in SGRs and AXPs neutron stars? Alternatively, could they be quark stars? vii) How many other manifestations of magnetars exist?

In order to answer to these open questions, more sensitive instruments, more detailed theories, and more data (probably in the next 30 years) are necessary.

A large diversity of neutron stars has been discovered by multifrequency observations from the radio band to the $\mathrm{X}$-ray and gamma-ray energy ranges. Among different manifestation of neutron stars - which include SGRs, AXPs, high-B pulsars (HBPs), high-E binaries (HEBs), rotating radio transients (RRATs), central compact objects (CCOs), rotation-powered radio pulsars (RPPs), and X-ray isolated neutron stars (XINSs) (Harding, 2013) - magnetars are the strongest magnetized objects.

These various manifestations of neutron stars show different characteristics of rotation period $\mathrm{P}$ and its derivative $\dot{\mathrm{P}}$. The measurements of $\mathrm{P}$ and $\dot{\mathrm{P}}$ provide to estimate the dipole magnetic field strength $\mathrm{B}_{\mathrm{d}}=3.2 \times 10^{19} \sqrt{\mathrm{P} \dot{\mathrm{P}}}$ and characteristic age $\tau_{\mathrm{c}}=\mathrm{P} / 2 \dot{\mathrm{P}}(\mathrm{e} . \mathrm{g}$. Manchester, 2017).

Figure 10 shows the P-P diagram (after Enoto, 2018), where SGRs and AXPs are collectively called "magnetars" since their slow rotation ( $\mathrm{P} \sim 2-12 \mathrm{~s}$ ) - with the exception of PSR J1119-6127 $(\mathrm{P}=0.41 \mathrm{~s})$ and PSR J1846-0258 $(\mathrm{P}=0.33 \mathrm{~s})-$ and high period derivatives $\left(\dot{\mathrm{P}} \sim 10^{-13}-10^{-9} \mathrm{~s}\right.$ $\mathrm{s}^{-1}$ ) indicate high magnetic fields $\mathrm{B}=10^{14-15} \mathrm{G}$ and young characteristic age $\tau_{\mathrm{c}} \lesssim 10-100 \mathrm{kyr}$. To date, there are 29 known magnetars in the Milky Way and local universe (see Table 2 of the paper by Giovannelli \& Sabau-Graziati, 2019).

It seems almost natural to think about a continuity among different classes of neutron star systems. However radio pulsations that have been observed from about 2000 neutron stars with weaker magnetic fields have never been detected from any of the known magnetars until the paper by Camilo et al. (2006) which showed that XTE J1810-197 - the first transient magnetar discovered (Ibrahim et al., 2004) - emits bright, narrow, highly linearly polarized radio pulses, observed at every rotation, thereby establishing that magnetars can be radio pulsars. Thus, these observations which link magnetars to ordinary radio pulsars, rule out alternative accretion models for AXPs, and provide a new window into the coronae of magnetars. 


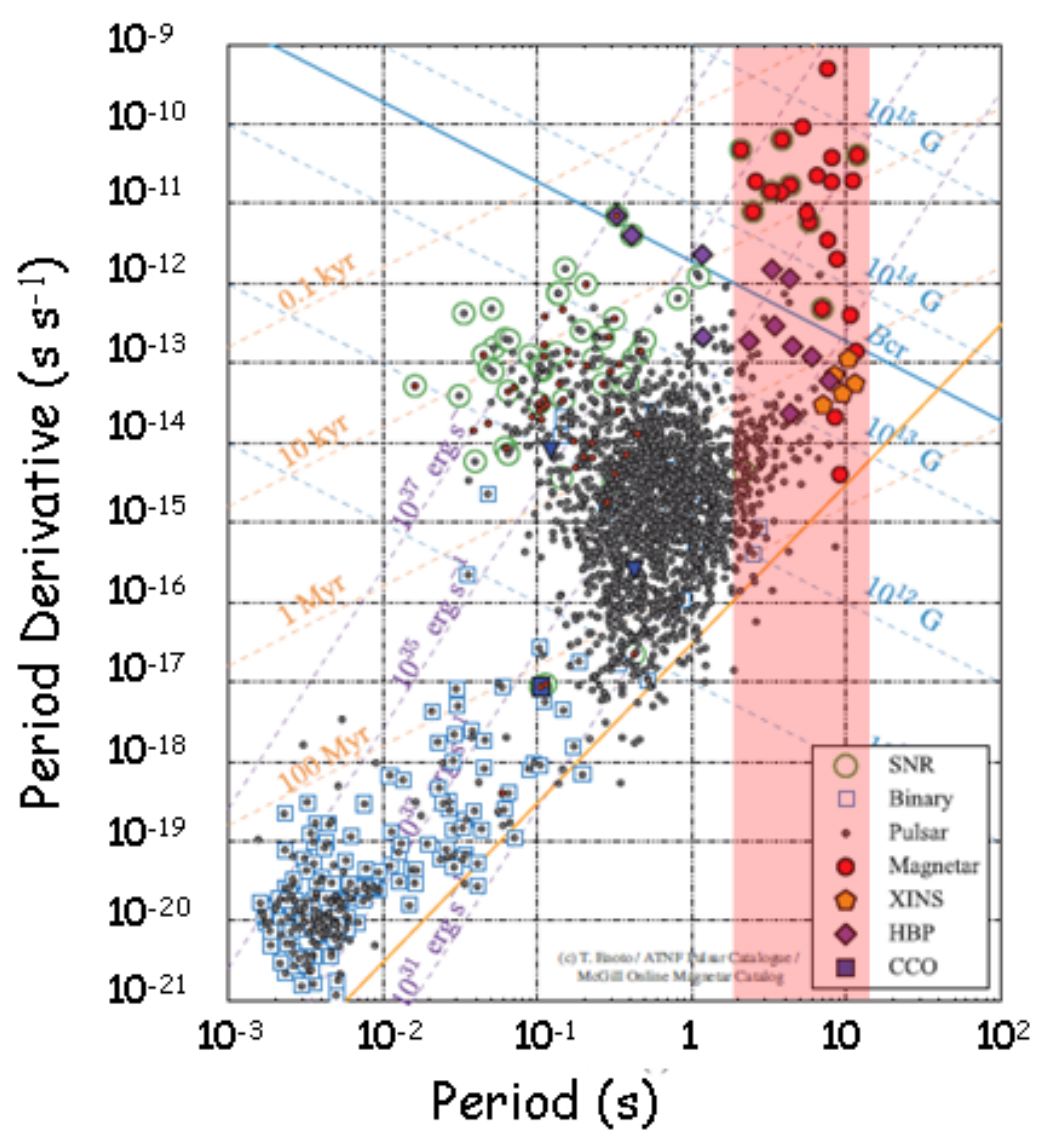

Figure 10: Magnetars and neutron stars on the $\mathrm{P}-\dot{\mathrm{P}}$ diagram. The light-red rectangle limits the range of magnetars periods (adapted from Enoto, 2018).

In the excellent review paper by Kaspi \& Beloborodov (2017) most of the critical points about magnetars have been deeply discussed. They concluded that: "The magnetar model has now been used to predict, naturally and uniquely, a wide variety of remarkable phenomena and behaviors in sources that once seemed highly anomalous. The now seamless chain of phenomenology from otherwise conventional radio pulsars through sources previously known for radically different behavior makes clear that these objects are one continuous family, with activity correlated with spin-inferred magnetic field strength. Recent advances in the physics of these objects, from the core through the crust and to the outer magnetosphere, hold significant promise.

Esposito, Rea \& Israel (2018) published a review on Some Sparse Considerations on Magnetars in which they remark their relevance for a vast range of different astrophysical topics, from the study of gamma-ray bursts and superluminous supernovae, to ultraluminous X-ray sources, fast radio bursts, and even to sources of gravitational waves.

Recently a surprise arrived from AR Sco, a white dwarf binary characterized by $\mathrm{P}_{\text {orb }}=3.57 \mathrm{~h}$, rotational period $\mathrm{P}_{\text {spin }}=1.97 \mathrm{~m}, 0.81 \mathrm{M}_{\odot}<M_{1} \lesssim 1.29 \mathrm{M}_{\odot}, 0.28 \mathrm{M}_{\odot}<M_{2} \lesssim 0.45 \mathrm{M}_{\odot}($ Marsh et al., 2016): it showed a strong linear polarization $(\lesssim 40 \%)$ variable with $\mathrm{P}_{\text {orb }}$ and $\mathrm{P}_{\text {spin }}$ and beat period (Buckley et al., 2017). The pulsed luminosity of AR Sco is powered by the spin-down of the rapidly-rotating WD which is highly magnetised $(\lesssim 500 \mathrm{MG})$. 
Figure 11 shows the position of AR Sco and AE Aqr in the diagram Log $L_{x}$ vs Log $\dot{E}$ (Buckley et al., 2017, after Becker \& Trümper, 1997).

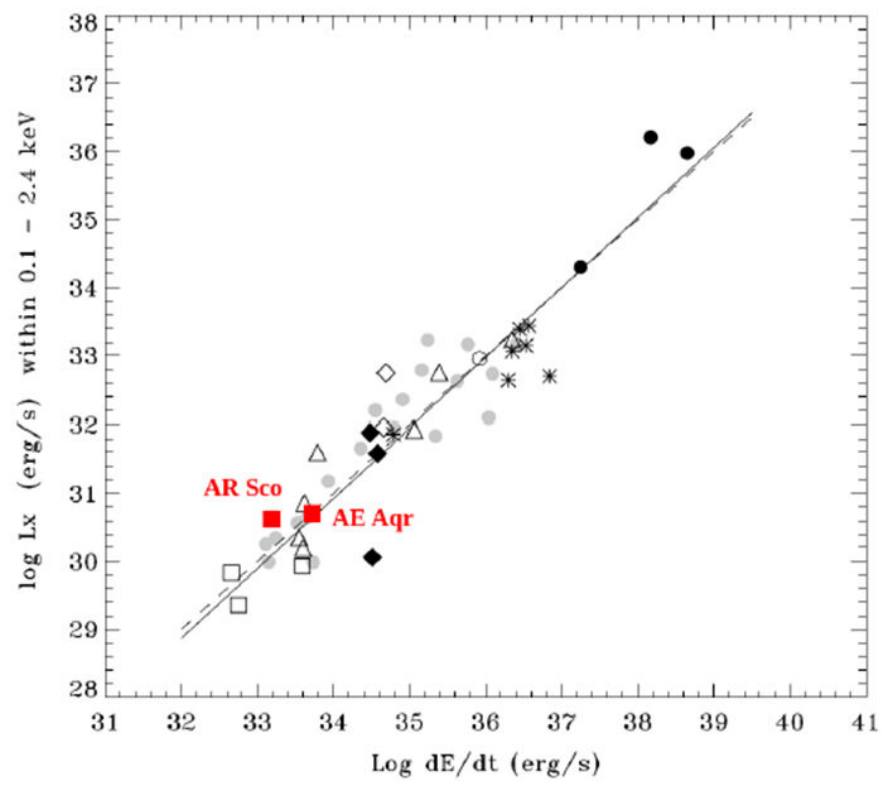

Figure 11: X-ray luminosity vs spin-down energy for all rotation-powered pulsar detected by ROSAT as of July 1997 (Becker \& Trümper, 1997). The positions of AR Sco and AE Aqr are superimposed (Buckley et al., 2017).

Thus, AR Sco is the first example of a white dwarf pulsar. This fact, in my opinion, open again the question about the origing of the magnetars: are they coming from white dwarf merger as suggested by e.g. Malheiro, Rueda \& Ruffini (2012), and Rueda et al. (2013)?

The discovery of a first white dwarf showing typical characteristics of pulsars, encourages the community of high energy physicists to undertake precise measurements to reveal other systems similar to AR Sco, which for reasons of evolutionary logic must exist.

The Scenario Machine (Monte Carlo simulations of binary evolution) permits to build up the complete picture of all possible evolutionary stages of binaries in the Galaxy. It was developed by Vladimir Lipunov and collaborators (Lipunov, 1987; Lipunov \& Postnov, 1988) and is useful obviously also in the case of the "newborn" white dwarf pulsar.

Indeed, just to illustrate the power of the Scenario Machine, Fig. 12 shows Lipunov's updated diagram, where the position of AR Sco is reported. It lies just in the ejector zone together with the pulsars.

In the following I will briefly discuss about the High Magnetic Field White Dwarfs (HMFWDs).

\subsection{High Magnetic Field White Dwarfs}

Magnetic fields are observed in main sequence stars and their white dwarf and neutron star progeny. The fields in these three groups of stars are likely to be linked via stellar evolution. However, stellar evolution calculations that include magnetic fields and rotation in a self consistent manner are only now beginning to be carried out, and then only with simplifying assumptions. 


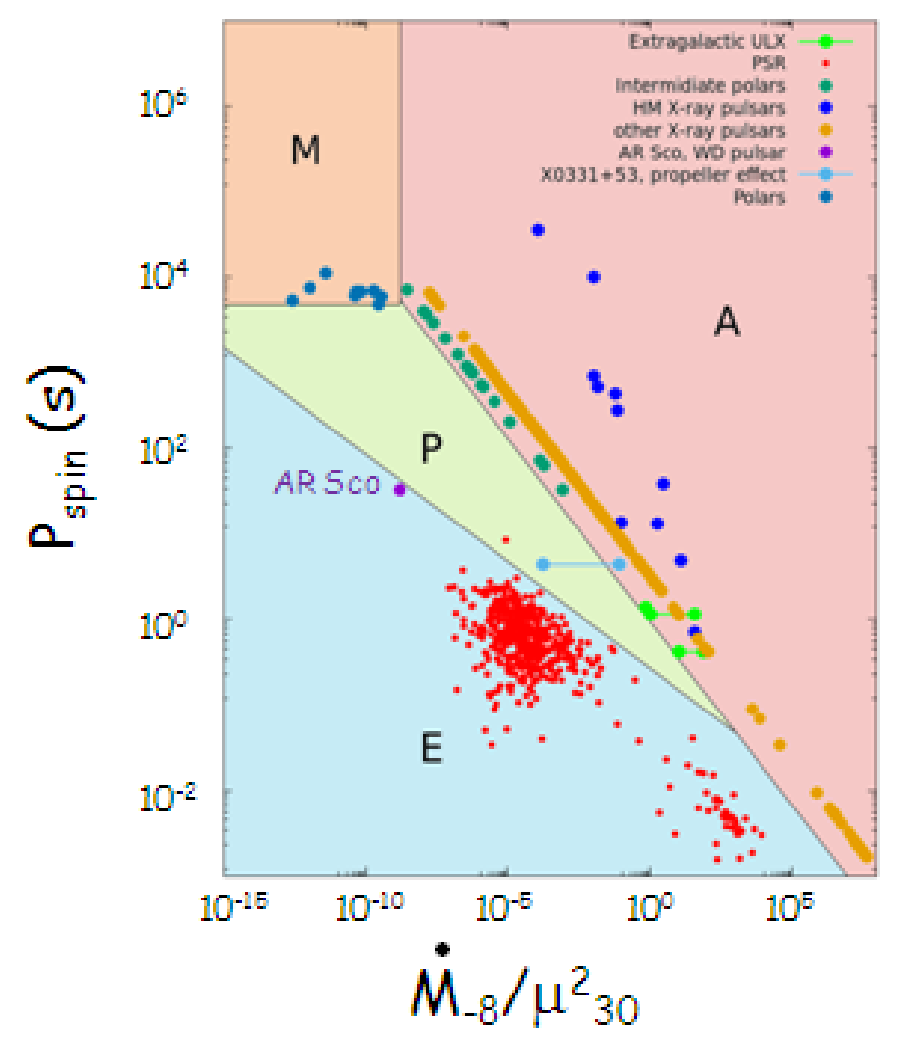

Figure 12: Lipunov's updated diagram from the scenario machine (adapted from Lipunov, 2018).

Some insights into the nature of magnetism of stars in these three groups can be obtained by a comparative study of their group properties (e.g. Ferrario \& Wickramasinghe, 2007).

While there is strong evidence that the magnetic fields in late type stars are dynamo generated, it is likely that the magnetic fields of stars on the upper main sequence are of fossil origin, perhaps dating back to the time of star formation.

We have the following approximate correspondences under flux conservation:

$$
\begin{aligned}
& \mathrm{B}_{\mathrm{B}-\mathrm{F}} \sim 300-30000 \mathrm{G} \Longrightarrow \mathrm{B}_{\mathrm{HFMWD}} \sim 10^{6}-10^{9} \mathrm{G} \\
& \mathrm{B}_{\mathrm{O}-\mathrm{B}} \sim ? ?-2000 \mathrm{G} \Longrightarrow \mathrm{B}_{\mathrm{NS}} \sim 10^{11}-10^{15} \mathrm{G}
\end{aligned}
$$

The lower field limit is still unknown for early B to O-type stars. A strong case can therefore be made for a fossil origin of fields at least at the high field end of the field distribution (Ferrario \& Wickramasinghe, 2007).

The rotational periods of WDs and NSs are scaling as $\mathrm{P}_{\mathrm{NS}} / \mathrm{P}_{\mathrm{WD}} \approx\left(\mathrm{R}_{\mathrm{NS}} / \mathrm{R}_{\mathrm{WD}}\right)^{2}$.

There are two possible origin of strong magnetic fields in WDs:

- The fossil field model (no stellar evolution models show how strong fossil magnetic flux can survive through the various stages of stellar evolution through to the WD phase).

- The merging star model (dynamo model of Wickramasinghe, Tout \& Ferrario, 2014): similar maximum magnetic fluxes may be expected for physical reasons if the fields are generated from differential rotation caused by merging). 
In the fossil scenario, the magnetic field strength $B$ scales with the radius of the star $R_{\star}$ as $B \propto$ $\mathrm{R}_{\star}{ }^{-2}$ (Ferrario, Melatos \& Zrake, 2015).

Figure 13 shows the distribution of rotation periods for magnetic white dwarfs (MWDs) (left panel) and neutron stars (NSs) (right panel) (adapted from Ferrario \& Wickramasinghe, 2005). Both diagrams have the same trend, except the scale factors of rotational periods $\mathrm{P}_{\text {rot }}$ (minutes for WDs, seconds for NSs) and magnetic field intensity ( $\sim 10^{7}-10^{9}$ for WDs and $\sim 10^{13}-10^{15} \mathrm{G}$ for NSs).

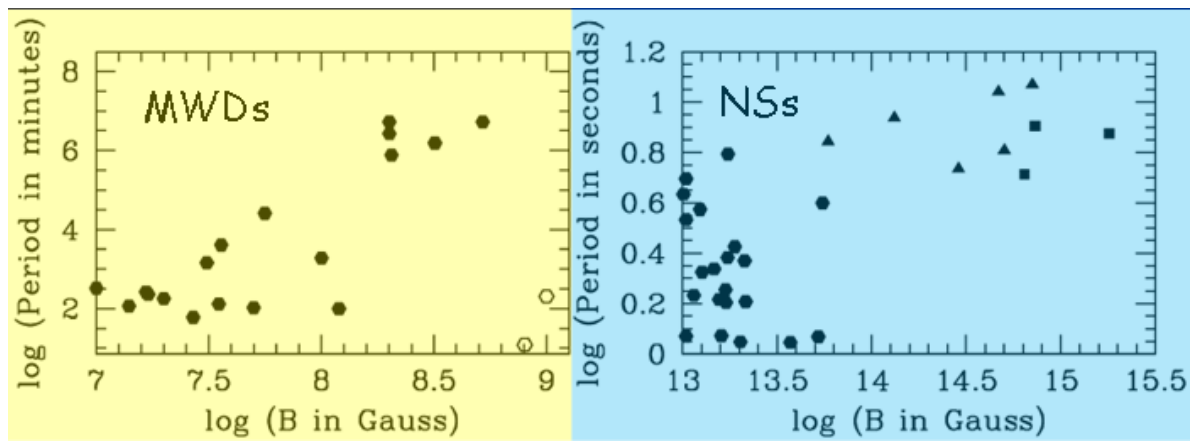

Figure 13: The distribution of rotation periods for magnetic white dwarfs (MWDs) (left panel) and neutron stars (NSs) (right panel) (adapted from Ferrario \& Wickramasinghe, 2005).

We still need to construct: (i) more realistic model atmospheres that allow for the presence of magnetic fields; (ii) stellar evolution tracks of intermediate mass stars that take into consideration both fossil and dynamo generated fields. Such calculations may be able to tell us whether all WDs are magnetic at some level.

The origin of fields in highly magnetic WDs is currently being debated. Although the newly proposed scenario that all high field MWDs (single and in binaries) are the result of close binary evolution and mergers is gaining momentum, the fossil field hypothesis cannot be totally dismissed (Ferrario, de Martino \& Gänsicke, 2015).

It is interesting to note in Fig. 14 the distribution of B for MCVs and MWDs (adapted from Ferrario, de Martino \& Gänsicke, 2015).

In MCVs there is an interesting relationship between the magnetic field strength and orbital period of the systems, as reported in Fig. 15 where the polars and IPs are separated by the blue line that marks the synchronization between orbital and spin periods of the cataclysmic systems (Giovannelli \& Sabau-Graziati, 2015a after Ferrario, de Martino \& Gänsicke, 2015).

Also the system AM CVn $\equiv$ HZ 29 with orbital period of 17.5 minutes, discovered by Smak (1967) was later recognized as a CV by Patterson (1992) and it constitutes the prototype of AM CVn stars - ultra-compact binaries - formed by a primary star WD, and a secondary degenerate or semi-degenerate star: e.g. white dwarf - white dwarf binaries $\left(\mathrm{P}_{\text {orb }}<80\right.$ minutes). White dwarf primaries and main sequence secondaries have binary orbital periods greater than 80 minutes.

Levitan (2013) in his PhD thesis about "AM CVn Systems with Palomar Transient Factory" updates the number of AM CVn stars. Figure 16 shows the number of CVs versus orbital period. It is clearly evident that $50 \%$ of CVs lie over the "period gap", $39 \%$ of $\mathrm{CVs}$ lie below the "period gap", and $11 \%$ within the "period gap" (indicated with the light blue rectangle). The place where 


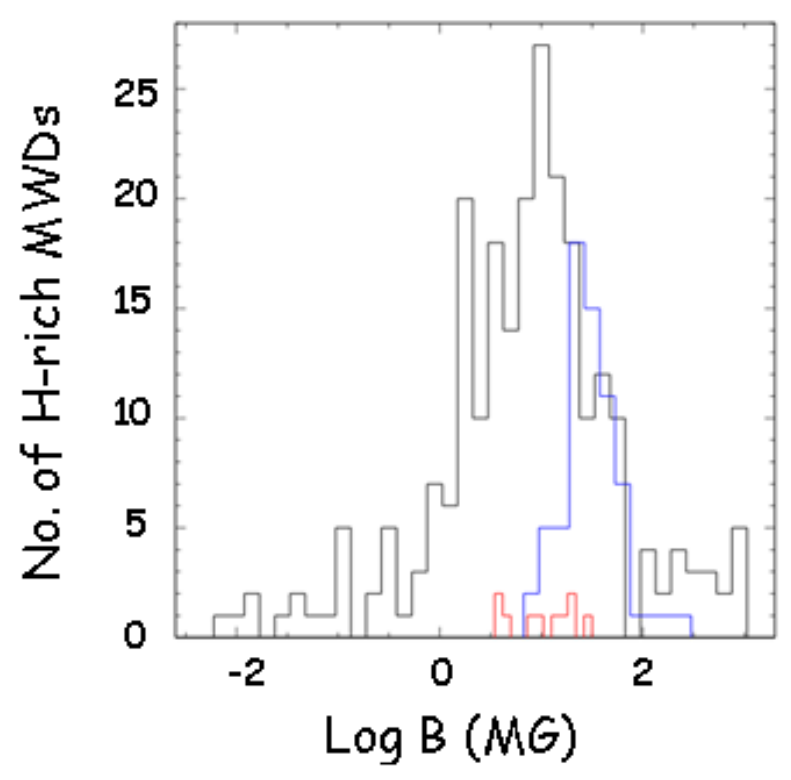

Figure 14: Distribution of magnetic field strength in polars (blue line), and IPs (red line) compared to that of single magnetic WDs (black line) (adapted from Ferrario, de Martino \& Gänsicke, 2015).

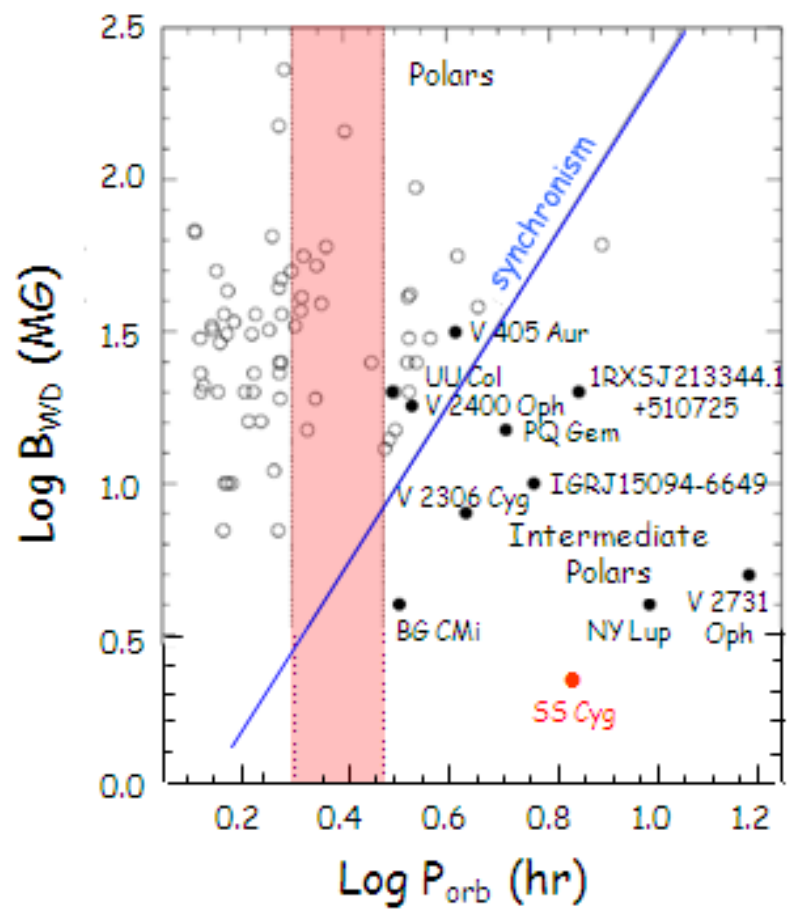

Figure 15: Magnetic field strength versus orbital period in MCVs. Polars and IPs are separated by the blue line that marks the border between systems with orbital period synchronized with the spin period (polars) and those without synchronization (IPs). Light red rectangle marks the so-called "period gap" (adopted from Giovannelli \& Sabau-Graziati, 2015a after Ferrario, de Martino \& Gänsicke, 2015). 


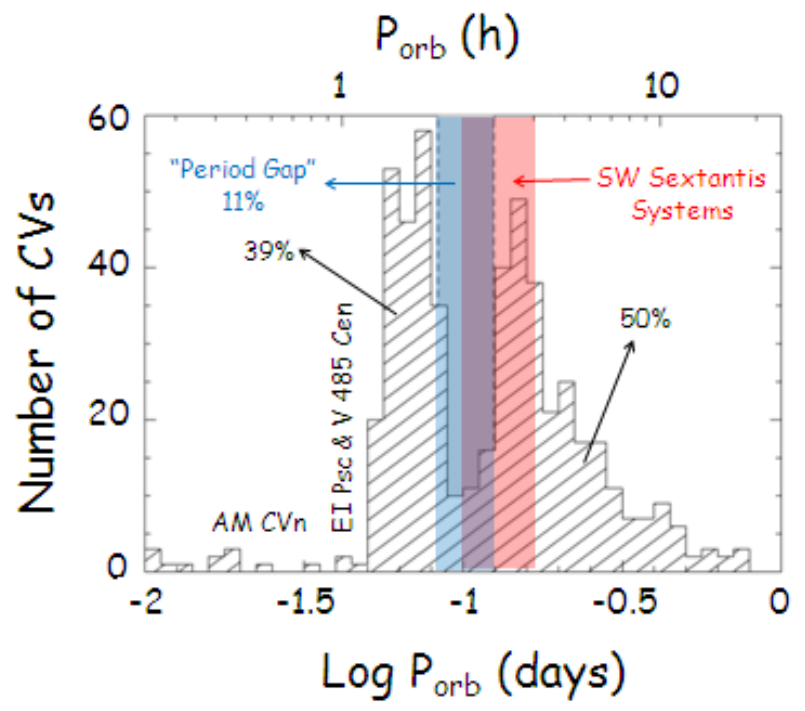

Figure 16: Number of CVs versus $P_{\text {orb }}$. Light blue and red rectangles mark the so-called "period gap" and the range in whicg SW Sex systems lie (adopted from Giovannelli \& Sabau-Graziati, 2015a after Gänsicke, 2005, and Levitan, 2013).

SW Sex systems lie (indicated with the light red rectangle) is partially in the "period gap". This demonstrates that the "period gap" does not exist. It appeared in the past like a gap because of lack of measurements and relatively low number of systems within the gap.

In my opinion is extremely important to remark once more that there is continuity among the "different classes" of CVs. This is coming from the pathway of evolution of CVs that is driven by angular momentum (J) loss. Orbital period $\left(\mathrm{P}_{\text {orb }}\right)$ decreases. All long $\mathrm{P}_{\text {orb }}$ CVs cross SW Sex regime before entering in the "Period Gap", where SW Sex systems lie. Thus, SW Sex phenomenon is an evolutionary stage in the life of CVs (Rodriguez-Gil, 2003; Schmidtobreick, 2013).

An exhaustive discussion about the state of art of the possible evolution of CVs including AM CVn systems is reported in the review paper by Giovannelli \& Sabau-Graziati (2015a) and references therein.

\subsection{Doppler tomography of accretion disks}

The fast Fourier Transform (FFT) in one, two, or three dimensions is an efficient algorithm well known for decades (see Cooley \& Tukey, 1965) as well as a traditional component of any serious numerical library. Three-dimensional (3D) FFT happens to be one of the most compute- and communication-intensive components in applications from a range of fields (for example, in turbulence, molecular dynamics, 3D tomography, and astrophysics), and for this reason there are many excellent parallel implementations in existence today. Pekurovsky (2012) introduced a popular software package called P3DFFT which implements FFT in three dimensions in a highly efficient and scalable way. This paper discussed P3DFFT implementation and performance in a way that helps guide the user in making optimal choices for parameters of their runs.

Horne (1985) and Marsh \& Horne (1988) developed Doppler tomography as a technique aimed at constructing a two-dimensional velocity image (tomogram) of the accretion disks of CVs using 
an emission line in its spectra sampled at a number of orbital phases. Doppler tomography has revolutionized the interpretation of orbitally phase-resolved spectroscopic observations of interacting binary systems. It has become a valuable tool for resolving the distribution of line emission in CVs and other binary systems. For instance Marsh (2001) in his review discussed the results of Doppler tomography applied to CVs. Outstanding successes to that date are the discovery of two-arm spiral shocks in cataclysmic variable accretion disks and the probing of the stream/magnetospheric interaction in magnetic cataclysmic variable stars. Doppler tomography has also told us much about the stream/disk interaction in non-magnetic systems and the irradiation of the secondary star in all systems.

Zhilkin \& Bisikalo (2009) described a 3D numerical code designed for simulations of magnetohydrodynamical (MHD) flows in semidetached binary systems. They found that even in case of a moderate magnetic field $\left(\mathrm{B}=10^{5} \mathrm{G}\right)$ the flow structure in the vicinity of the accretor of a $\mathrm{CV}$ was drastically changed and the funnel flows were formed in the system.

Bisikalo \& Zhilkin (2012) discussed the Flow Structure in Magnetic CVs by studying the physical processes which go on in magnetic CVs with the mass transfer between the components. Using results of 3D MHD simulations, they investigated variations of the main characteristics of accretion disks depending on the value of the magnetic induction on the surface of the accreting star. Zhilkin, Bisikalo \& Mason (2012) discussed on "Full 3D MHD calculations of accretion flow structure in magnetic cataclysmic variables with strong, complex magnetic fields".

Bisikalo et al. (2008) discussed Dopppler tomography of the CV SS Cygni in quiescence. Boneva et al. (2009) discussed the Doppler mapping in the case of SS Cygni in outburst and finally Kononov et al. (2012) used Doppler tomography for studying the pre-outburst accretion disk of SS Cygni.

Zhilkin \& Bisikalo (2011) presented a 3D numerical model to simulate plasma flows in close binary systems with accretors having strong proper magnetic fields. The model is based on an assumption that plasma dynamics is determined by a slow average flow against a background of propagating MHD waves. Equations describing the slow plasma motion are obtained as a result of the corresponding averaging procedure. The model takes into account the magnetic diffusivity which is due to the current dissipation in turbulent vortexes, magnetic buoyancy and wave MHD turbulence. They presented results of the simulations for MHD flows in a polar-like binary system. Results of the simulations show that in semi-detached binary systems with strong magnetic fields $\left(\mathrm{B}=10^{7} \mathrm{G}\right)$ the flow structure can differ sufficiently from that of systems with moderate magnetic fields $\left(B=10^{5} \mathrm{G}\right)$. Accretion disks in such magnetic systems systems $\left(B=10^{7} \mathrm{G}\right)$ are not formed and the flow is collimated into the accretion stream starting from the inner Lagrange point $\mathrm{L}_{1}$ and finishing on the magnetic poles of the accretor. This flow structure corresponds to the case of polars.

Kotze, Potter \& McBride $(2015,2016)$ explored inside-out Doppler tomography for nonmagnetic and magnetic cataclysmic variables, respectively. The code they used is available at http://www.saao.ac.za/ ejk/doptomog/main.html.

Doppler tomography has been applied also to fusion plasmas giving an image of the fastion velocity distribution function in the tokamak ASDEX Upgrade (Salewski et al., 2015). It is interesting to note that Bisikalo et al. (2016) presented a method that can be used to recover the spectrum of turbulence from observations of optically thin emission lines formed in astrophysical 
disks. Within this method, they analyze how line intensity fluctuations depend on the angular resolution of the instrument, used for the observations. The method allows us to restore the slope of the power spectrum of velocity turbulent pulsations and estimate the upper boundary of the turbulence scale.

Doppler tomography is very useful also for analyzing the asymmetric MHD outflows/jets from accreting T Tauri stars, and even in planetary science, as discussed by Giovannelli \& Sabau-Graziati (2016a, and the references therein).

\section{Classical and Recurrent Novae}

A fundamental review about Theory and Observations of Classical Novae appeared long time ago, but it is still important also because of an exhaustive historical excursion on classical novae (CNe) by Gallagher \& Starrfield (1978).

Starrfield \& Sparks (1987) discussed about a new class of CNe in which the outbursts occur on $\mathrm{ONeMg}$ white dwarfs in close binary systems in contrast to the other outbursts which occurr on $\mathrm{CO}$ white dwarfs. Theoretical simulations of this class have been found to be in excellent agreement with the observations.

A detailed discussion about The Nature of Classical Novae Outbursts can be found in Truran (2002), and references therein.

Classical novae are expected to recur on timescales from 100,000 years to just a few decades. The most important physical parameters controlling this recurrence timescale are the WD mass, and the mass accretion rate from the secondary (e.g. Yaron et al. 2005). Once classical nova (CN) is recorded more than once, it can be designated as "recurrent" (RN). Since the WD and the binary system remain intact after an outburst, it is possible that classical novae may actually be the same as recurrent novae if observed over a long enough time period. While the interval between outbursts of recurrent novae range from 10 to 100 years, it has been estimated that the time interval for classical novae would range from about 30,000 years for a $1.3 \mathrm{M}_{\odot}$ WD to 100,000 years for a $0.6 \mathrm{M}_{\odot} \mathrm{WD}$. Given long enough - it is expected that all classical novae will be observed as recurrent novae.

The long term behaviour of classical old novae, and the optical behaviour of $\mathrm{CNe}$ in outburst were discussed by Bianchini (1990), and Seitter (1990), respectively. The books by Cassatella \& Viotti (1990) and by Bode \& Evans (2008) are very useful for studying the physics of classical novae.

Recurrent novae are a rare sub-class of CVs; WDs accreting material from a binary companion in which more than one classical nova-type outburst has been observed (see the book of Hellier, 2001 for a comprehensive review of CVs). Nova outbursts are suspected to be due to a thermonuclear runaway on the surface of the WD, which releases huge amounts of thermal energy once a critical pressure is reached at the base of the shell of accreted material.

One of the most interesting RNe is RS Ophiuchi (RS Oph). It is an amazingly prolific recurrent nova, with recorded outbursts in 1898, 1907, 1933, 1945, 1958, 1967, 1985 and 2006 (Schaefer 2010). The short time between outbursts ( $\sim 20$ yrs) suggests that RS Oph hosts a massive WD accreting material at a significantly high rate.

In the latter paper Schaefer discussed not only RS Oph, but also the photometric histories of all known galactic RNe. 
Classical and recurrent nova outbursts have been discussed by Bode (2010, 2013) and Evans (2011). The proceedings of a conference about RS Oph and recurrent phenomenon can be very useful for details (Evans et al., 2008). General properties of quiescent novae have been discussed by Warner (2002). The very useful book of Bode \& Evans (2008) about classical novae examines thermonuclear processes, the evolution of nova systems, nova atmospheres and winds, the evolution of dust and molecules in novae, nova remnants, and observations of novae in other galaxies. It includes observations across the electromagnetic spectrum, from radio to gamma rays, and discusses some of the most important outstanding problems in classical nova research.

Of the $\sim 400$ known Galactic classical novae, only 10 of them are recurrent. Eight of them harbor evolved secondary stars, contrary to classical novae that contain main sequence stars (Darnley et al., 2011). They propose a new nova classification based on the evolutionary state of the secondary star, contrary the current schemes based on the properties of outbursts. Such classification contains three groups of novae: i) Main Sequence Nova (MS-Nova); ii) Sub-Giant Nova (SG-Nova); and iii) Red Giant branch Nova (RG-Nova).

An important not yet resolved problem is connected with the evolution and fate of Classical Novae. Patterson (2014) discussed this crucial problem. Classical novae rise from obscurity to shine among the brightest stars in the Galaxy. The story of how they return to quiescence is still only dimly known. Vast amounts of energy are loosed upon the WD and its companion, and the light curves of post-novae suggest that they take not a few years, but a few thousand years, to return to quiescence. In the meantime, the secondary may experience a lot of heating from the WD's radiation - enough to overwhelm its intrinsic nuclear luminosity. For this purpose he mentioned the case of BK Lyncis - the oldest old nova and a bell-wether for CVs evolution (Patterson et al., 2013). They discussed stellar physics behind this suggestion and proposed how it might be tested by time-series photometry in the months and years (and if possible, centuries) after outburst.

RNe play an important role in the studies of SN Ia progenitors (Surina, Bode \& Darnley, 2013). RNe are likely progenitors of Type-Ia supernovae.

On the contrary, Shafter et al. (2015) estimated that $\sim 4 \%$ of the nova eruptions seen in M31 over the past century are associated with RNe. A Monte Carlo analysis shows that the discovery efficiency for RNe may be as low as $10 \%$ that for novae in general, suggesting that as many as one in three nova eruptions observed in M31 arise from progenitor systems having recurrence times $\lesssim 100$ yr. For plausible system parameters, it appears unlikely that RNe can provide a significant channel for the production of Type-Ia supernovae.

Important works have been developed about extragalactic nova populations (Shafter et al., 2014). Nova rates have been measured for more than a dozen galaxies spanning a wide range of Hubble types. They found that the recurrent nova population in the LMC appears to be higher than that seen in M31 and the Galaxy.

In order to brave this important problem the use of archival data is the only way to answer the big question. Now, huge and comprehensive set of archival RN data go back to 1890 .

Excellent work about the archival data has been promoted by René Hudec $(2015,2017)$, who scanned thousands plates belong to numerous astronomical observatories spread in the whole world (e.g. Hudec, R. \& Hudec, L., 2013). Indeed, the astronomical plate archives represent the only method how to study the behavior of the CVs (and other astrophysical objects in general) over very long (100 years or even more) time intervals, and the only method to go back in time. In addition, 
huge monitoring times (up to 30,000 hrs of continuous monitoring) are available allowing to detect and to study rare events such as outbursts. The databases allow to study prominent spectra and/or spectral changes as well (Hudec, R. Hudec, L. \& Klíma, M., 2012).

Starrfield, Iliadis \& Hix (2016) in their review about The Thermonuclear Runaway and the Classical Nova Outburst described both the recent advances in our understanding of the progress of the outburst and outline some of the puzzles that are still outstanding. They reported on the effects of improving both the nuclear reaction rate library and including a modern nuclear reaction network in their one-dimensional, fully implicit, hydrodynamic computer code. In addition, there has been progress in observational studies of SNe Ia with implications about the progenitors, and they discussed that in their review.

In a review about galactic and extragalactic novae Poggiani (2017a) discussed the multifrequency observations that are contributing to understanding the process of explosions and of the long-term evolution. She discussed the observations of novae over the electromagnetic spectrum, focusing on the morphology of the decline light curves, the spectroscopic investigations, the long term evolution, the recurrent novae, the gamma ray emission in novae, extragalactic novae, and the possible gravitational-wave emission of novae. An update of this review can be found in Poggiani (2019).

\section{Progenitors of Type-Ia Supernovae}

It is well accepted by the community that Type-Ia SNe are the result of the explosion of a carbon-oxygen WD that grows to near Chandrasekhar's limit in a close binary system (Hoyle \& Fowler, 1960). But the debate is focussed around the different kinds of progenitors. Indeed, in the past, two families of progenitor models have been proposed. They differ in the mode of WD mass increase. The first family is the so-called single degenerate (SD) model (Whelan \& Iben, 1973), in which the WD accretes and burns hydrogen-rich material from the companion. The second family is the so-called double degenerate (DD) model, in which the merging of two WDs in a close binary triggers the explosion (Webbing, 1984; Iben \& Tutukov, 1984). The two scenarios produce different delay times for the birth of the binary system to explosion. Thus it is hopefully possible to discover the progenitors of Type-Ia SNe by studying their delay time distribution (DTD). The DTD can be determined empirically from the lag between the cosmic star formation rate and Type-Ia SN birthrate.

Maoz \& Mannucci (2012) reviewed the progress in measuring SN ia rates in various environments and redshifts, and their use to reconstruct the SN Ia delay-time distribution DTD - the SN rate versus time that would follow a hypothetical brief burst of star formation. A good number of DTD measurements, using a variety of methods, appear to be converging. At delays $1<\mathrm{t}<10$ Gyr, these measurements show a similar, $\sim \mathrm{t}^{-1}$, power-law shape. The DTD peaks at the shortest delays probed. This result supports the idea of a DD progenitor origin for SNe Ia. SD progenitors may still play a role in producing short-delay SNe Ia, or perhaps all SNe Ia, if the red-giant donor channel is more efficient than is found by most theoretical models. The DTD normalization enjoys fairly good agreement (though perhaps some tension), among the various measurements, with a Hubble time-integrated DTD value of about $2 \pm 1$ SNe Ia per $1000 \mathrm{M}_{\odot}$ (stellar mass formed with a low-mass turnover initial mass function). The local WD binary population suggests that the WD 
merger rate can explain the Galactic SN Ia rate, but only if sub-Chandra mergers lead to SNIa events. They pointed to some future directions that should lead to progress in the field, including measurement of the bivariate (delay and stretch) SN Ia response function.

Maoz, Mannucci \& Nelemans (2014) discussed in their review on Observational Clues to the Progenitors of Type Ia Supernovae the significant observational progress in addressing the progenitor problem. The observational results are not yet conclusive and sometimes prone to competing theoretical interpretations. Nevertheless, it appears that DD progenitors long considered the underdog option, could be behind some, if not all, SNe Ia.

Wang et al. (2013) introduced their binary evolution models of various progenitor scenarios. They obtained many properties of the surviving companions from various SD systems, which may be helpful for identifying SN Ia progenitor model. They conclude tha it seems likely that more than one progenitor model may be required to explain the observed diversity of SNe Ia.

The energy released through runaway thermonuclear process ejects the majority of the unburnt hydrogen from the surface of the star in a shell of material moving at speeds of up to $1.5 \times 10^{3} \mathrm{~km}$ $\mathrm{s}^{-1}$. This produces a bright but short-lived burst of light - the nova.

Although Type-Ia supernovae appear to have similar origin to classical novae, there are key differences. The most important is that in a classical nova, the thermonuclear runaway occurs only on the surface of the star, allowing the WD and the binary system to remain intact (e.g. Townsley \& Bildsten, 2005). In a Type-Ia supernova, the thermonuclear runaway occurs within WD itself, completely disrupting the progenitor. This is reflected in the amount of energy released in the explosions, with classical novae releasing $\sim 10^{44} \mathrm{erg}$, and Type-Ia supernovae $\sim 10^{51} \mathrm{erg}$.

The possible progenitors of SN Ia are: i) Recurrent Novae; ii) Symbiotic stars; iii) Super-soft sources; iv) Double WD Binaries; and v) WDs accreting material from red-giant companions.

i) Recurrent Novae are just a subset of ordinary novae that happen to go off more than once per century.

As such, they are binary systems with matter flowing off a companion star onto a WD, accumulating on its surface until the pressure gets high enough to trigger a thermonuclear runaway that is the nova.

Only 10 RNe are known in our Milky Way galaxy, including: U Sco $(1863,1907,1917,1936$, 1945, 1969, 1979, 1987, 1999); Т Pyx (1890, 1902, 1920, 1944, 1967, 2011); Т CrB (1866, 1946); RS Oph (1898, 1907, 1933, 1945, 1958, 1967, 1985, 2006). For a deep discussion see the Doctoral Dissertation on Recurrent Novae and Type Ia Supernova Progenitors by Ashley Sara Pagnotta (2012).

To recur with $\tau_{\text {rec }}<100$ years, RNe must have: high WD mass $\left(1.2 \mathrm{M}_{\odot}<\mathrm{M}_{\mathrm{WD}}<\mathrm{M}_{\text {Chandra }}\right)$, and high accretion rate $\left(\dot{\mathrm{M}} \sim 10^{-7} \mathrm{M}_{\odot} \mathrm{yr}^{-1}\right)$. SN Ia occurs if: i) the mass ejected for each eruption is less than the mass accreted onto the WD $\left(\mathrm{M}_{\text {ejected }}<\dot{\mathrm{M}} \tau_{\text {rec }}\right)$; ii) the rate of death RNe must be enough to produce the $S N$ Ia rate $\left(R_{R N d e a t h}=R_{S N I a}\right)$, being $R_{R N d e a t h}=N_{R N} \times\left(0.2 M_{\odot} \dot{M}\right)$.

In order to solve the problems we need to know $\tau_{\text {rec }}$ (recurrence time scale) from archive plates, $\mathrm{N}_{\mathrm{RN}}$ (number of RNe in the Milky Way) from archive plates and AAVSO, $\dot{\mathrm{M}}$ (mass accretion rate onto WD) from the average in the last century, $\mathrm{M}_{\mathrm{ejected}}$ (mass ejected in eruption) from pre-eruption eclipse timing.

Some results have been obtained for becoming optimists in solving the problem of SN Ia production. Indeed Schaefer (2011) obtained for CI Aql and U Sco $\mathrm{M}_{\text {ejected }}<<\dot{\mathrm{M}} \tau_{\text {rec }}$ ). 
Thus, WDs are gaining mass and the latter RNe will collapse as SN Ia. Moreover, for the Milky Way, M31, and LMC $\mathrm{R}_{\mathrm{RNdeath}} \sim \mathrm{N}_{\mathrm{RN}}$. Then there are enough RNe to supply the Type-Ia $\mathrm{SN}$ events.

ii) Symbiotic Stars contain WDs efficiently accreting material from the secondary star. In most cases they steadily burn H-rich material allowing them to grow in mass. Some of these systems can produce high mass WDs. In symbiotic RNe (SyRNe) the WD mass is already very close to Chandrasekhar's limit. For instance in V 407 Cyg a very massive WD is accreting material at a rate of $\sim 10^{-7} \mathrm{M}_{\odot} \mathrm{yr}^{-1}$ from a Mira-type companion (Mikołajewska, 2013).

iii) Super-soft Sources are probably WDs that accrete material and burn hydrogen. Voss \& Nelemans (2008) discovered an object at the position of the Type-Ia SN2007on in the elliptical galaxy NGC1404 on pre-supernova archival X-ray images. This result favours the accretion model (SD) for this supernova, although the host galaxy is older than the age at which the explosions are predicted in SD models. However, the DD model cannot be ruled out by this event because a hot accretion disc is probably the intermediate configuration of the system, between first WD-WD Roche-lobe contact and explosion (Yoon, Podsiadlowski \& Rosswog, 2007).

Greggio, Renzini \& Daddi (2008) starting from the fact that Type-Ia SN events occur over an extended period of time, following a distribution of delay times (DDT), discussed theoretical DDT functions that accommodate both "prompt" and 'tardy" SN events derived by empirically-based DDT functions. Moreover such theoretical DDT functions can account for all available observational constraints. The result is that SD/DD mix of SNIa's is predicted to vary in a systematic fashion as function of cosmic time (redshift).

Di Stefano (2010) discussed about the possible progenitors of SNe Ia. In a canonical model, the progenitors of SNe Ia are accreting, nuclear-burning white dwarfs (NBWDs), which explode when the white dwarf reaches the Chandrasekhar mass, $\mathrm{M}_{\mathrm{C}}$. Such massive NBWDs are hot $(\mathrm{kT} \sim$ $100 \mathrm{eV}$ ), luminous $\left(\mathrm{L} \sim 10^{38} \mathrm{erg} \mathrm{s}^{-1}\right.$ ), and are potentially observable as luminous supersoft X-ray sources (SSSs). During the past several years, surveys for soft X-ray sources in external galaxies have been conducted. In his paper Di Stefano shows that the results falsify the hypothesis that a large fraction of progenitors are NBWDs which are presently observable as SSSs. The data also place limits on sub-M $\mathrm{M}_{\mathrm{C}}$ models. While SN Ia progenitors may pass through one or more phases of SSS activity, these phases are far shorter than the time needed to accrete most of the matter that brings them close to $\mathrm{M}_{\mathrm{C}}$.

iv) Double WDs Binaries are systems containing two WDs that can merge and giving rise to SN explosion. Yoon, Podsiadlowski \& Rosswog (2007) explored the evolution of the merger of two carbon-oxygen (CO) WDs. Their results imply that at least some products of double CO WDs merger may be considered good candidates for the progenitors of Type-Ia SNe. Brown et al. (2011) and Kilic et al. (2011) studied a complete colour-selected sample of double-degenerate binary systems containing extremely low mass (ELM) $\left(\leq 0.25 \mathrm{M}_{\odot}\right)$ WDs. Milky Way disc ELM WDs have a merger rate of $\approx 4 \times 10^{-5} \mathrm{yr}^{-1}$ due to gravitational wave radiation. The ELM WD systems that undergo stable mass transfer can account for about $3 \%$ of AM CVn stars. The most important fact is that the ELM WD systems that may detonate merge at a rate comparable to the estimate rate of underluminous $\mathrm{SNe}$. These $\mathrm{SNe}$ are rare explosions estimated to produce only $\sim 0.2 \mathrm{M}_{\odot}$ worth of ejecta. At least $25 \%$ of ELM WD sample belong to the old tick disc and halo components of our Galaxy. Thus, if merging ELM WD systems are the progenitors of under- 
luminous $\mathrm{SNe}$, transient surveys must find them in both elliptical and spiral galaxies.

v) WDs accreting material from red-giant companions. Observations carried out by Patat et al. (2008) with VLT-UVES allowed to detect circumstellar material in a normal Type-Ia SN. The expansion velocities, densities and dimensions of the circumstellar envelope indicate that this material was ejected from the system prior to the explosion. The relatively low expansion velocities favour a progenitor system where a WD accretes material from a companion star, which is in the red-giant phase at the time of explosion.

However, the explosion of the SN 1987A in the Large Magellanic Cloud (LMC) was the first naked-eye supernova since Kepler's supernova in 1604. Neutrino detections from the event dramatically confirmed the long-held belief that this type of supernova is triggered by the final collapse of the core of a massive star, but in many other respects it was a very unusual, even anomalous event. Indeed, the progenitor was a blue supergiant instead of a red supergiant, as had been predicted theoretically (Podsiadlowski, Morrisa \& Ivanovac, 2007), and the system was surrounded by a spectacular triple-ring nebula that consists of material that was ejected only 20,000 years before the explosion (Podsiadlowski, 1991). Podsiadlowski (2017) discussed the mystery of the supernova progenitor and how all the evidence points toward a dramatic event that occurred some 20,000 years ago, the merger of two massive stars and how future observations will further help to prove or refute the current picture. Thus, this event opened a crisis in the comprehension of the progenitors that with their collapse trigger the SN Ia explosions.

Bianco et al. (2011) searched for a signature of a non-degenerate companion in three years of Supernova Legacy Survey data. They found that a contribution from WD/red-giant binary system to Type-Ia SN explosions greater than $10 \%$ at $2 \sigma$, and than $20 \%$ at $3 \sigma$ level is ruled out.

Type-Ia SNe are used as primary distance indicators in cosmology (e.g. Phillips, 2005). Phillips (2012) reviewed the near-infrared (NIR) of Type-Ia SNe concluding that such SNe are essentially perfect standard candles in the NIR, displaying only a slight dependence of peak luminosity on decline rate and colour. Lira (1995) first noted that B-V evolution during the period from 30 to 90 days after V maximum is remarkably similar for all SN Ia events, regardless of light-curve shape. This fact was used by Phillips et al. (1999) to calibrate the dependence of the $B_{\max }-V_{\max }$ and $\mathrm{V}_{\max }-\mathrm{I}_{\max }$ colours on the light curve parameter $\Delta \mathrm{m}_{15}$ (B) which can, in turn, be used to separately evaluate the host galaxy extinction. Using these methods for eliminating the effect of the reddening, they reanalyzed the functional form of the decline rate versus luminosity relationship and gave a value of the Hubble constant of $\mathrm{H}_{0}=63.3 \pm 2.2 \pm 3.3 \mathrm{~km} \mathrm{~s}^{-1} \mathrm{Mpc}^{-1}$.

The use of Type-Ia SNe is also fundamental for determining some cosmological constraints, such as $\Omega_{\mathrm{M}}$ and $\Omega_{\Lambda}$ that fit a $\Lambda$ CDM models with values of $0.211 \pm 0.034$ (stat) \pm 0.069 (sys) using a set of 252 high-redshift SNe (Guy et al., 2010) and $0.713_{-0.029}^{+0.027}$ (stat) ${ }_{-0.039}^{+0.036}$ (sys) using a set of low-redshift nearby-Hubble-flow SNe (Kowalski et al., 2008), respectively.

In order to explore the difficult topic of the expansion of the Universe it is necessary to know the evolution of metallicity in old Universe that changes the Hubble Diagram shape. The proposed space observatory Super Nova Acceleration Probe (SNAP) was designed to measure the expansion of the Universe and to determine the nature of the mysterious Dark Energy that is accelerating this expansion (Aldering, 2005). 
In August 2010, the Board on Physics and Astronomy of the National Science Foundation recommended the Wide Field Infrared Survey Telescope (WFIRST) mission - renamed as "Nancy Grace Roman Space Telescope" to honor a NASA pioneer -, a renamed JDEM-Omega proposal (Stril, Cahn \& Linder, 2010) which has superseded SNAP, Destiny, and ADEPT, as the highest priority for development in the decade around 2020. This would be a 1.5-meter telescope with a 144-megapixel HgCdTe focal plane array, located at the L2 Lagrange point. Nancy Grace Roman Space Telescope will have a panoramic field of view of the Universe that is 100 times wider than Hubble Space Telescope, and it is designed to settle essential questions in the areas of dark energy, exoplanets, and infrared astrophysics (https://roman.gsfc.nasa.gov/).

The research about the progenitors of SN Ia is of course one of the most important problems, since it is strictly connected with the evolution of CVs. For instance, Maguire et al. (2012) present an analysis of the maximum light, near-ultraviolet (NUV; $2900<\lambda<5500$ ) spectra of 32 lowredshift $(0.001<\mathrm{z}<0.08)$ SNe Ia, obtained with the Hubble Space Telescope (HST) using the Space Telescope Imaging Spectrograph. They combine this spectroscopic sample with high-quality gri light curves obtained with robotic telescopes to measure SN Ia photometric parameters, such as stretch (light-curve width), optical colour and brightness. They confirm and strengthen earlier conclusions regarding the complex behaviour of SNe Ia in the NUV spectral region, but suggest that the correlations found are more useful in putting tighter constraints on the progenitor systems of SNe Ia and how their progenitor channels may vary with host galaxy properties (e.g. metallicity: Kistler et al., 2013) rather than improving the use of SNe Ia as cosmological probes.

Wang \& Han (2012) reviewed SD and DD models for explaining the possible different progenitors. They concluded that it seems likely that more than one progenitor model, including some variants of the SD and DD models, may be required to explain the observed diversity of SNe Ia.

Darnley et al. (2014) discussed on the galactic nova progenitor population. They presented a selection of the work and rationale that led to the proposal of a new nova classification scheme based not on the outburst properties but on the nature of the quiescent system. They also outlined the results of a photometric survey of a sample of quiescent Galactic novae, showing that the evolutionary state of the secondary can be easily determined and leading to a number of predictions, including their relevance to extragalactic work and the proposed link to type-Ia SNe.

In order to solve the problem of determining the SN Ia progenitors, it is also important to look at the $\mathrm{RNe}$ that show many similarities to $\mathrm{CNe}$, but have had more than one recorded outburst. $\mathrm{RNe}$ play an important role as one of the suspected progenitor systems of Type-Ia SNe, which are used as primary distance indicators in cosmology. Thus, it is important to investigate the nature of their central binary systems to determine the relation between the parameters of the central system and the outburst type, and finally ascertain the population of novae that might be available to give rise to the progenitors of Type-Ia SNe. Surina, Bode \& Darnley (2015) adopted a low outburst amplitude as a criterion that may help distinguish $\mathrm{RNe}$ from $\mathrm{CNe}$ and was therefore used to select targets for observations from ground-based observatories including the Liverpool Telescope and the Southern African Large Telescope as well as the full-sky space-based archive of the Solar Mass Ejection Imager (SMEI). They found that at least four objects currently classified as $\mathrm{CNe}$ are possibly RNe candidates based on their quiescent spectra. They also searched the SMEI archive for additional outbursts of bright $\mathrm{CNe}$ that might otherwise have been missed but did not find a conclusive example. 
Another possible channel for triggering the explosion of SN Ia is that discussed by Chiosi et al. (2015). They explore the possibility that isolated CO-WDs with mass smaller than the Chandrasekhar limit may undergo nuclear runaway and SN explosion. If this channel could be confirmed it should be possible (i) to explain the star formation rate dependence of the SN Ia rate (e.g. Mannucci, Della Valle \& Panagia, 2006); (ii) to provide some clues to interpreting the observational data on the ejected mass distribution of type-Ia SNe showing a significant rate of non-Chandrasekhar-mass progenitors of mass as low as $0.8 \mathrm{M}_{\odot}$ (Scalzo, Ruiter \& Sim, 2014); and (iii) to account for the SNe exploding inside Planetary Nebulae in alternative to the core-degenerate scenario in which a WD merges with the hot core of an AGB star on a time interval $\leq 10^{8} \mathrm{yr}$ since the WD formation (see Tsebrenko \& Soker, 2015, for more details). With the models of Chiosi et al. (2015), a single CO-WD may reach the explosion stage soon after the formation if sufficiently massive $\left(>1.0 \mathrm{M}_{\odot}\right)$ and sufficiently rich in residual hydrogen $\left(\mathrm{X}_{\mathrm{H}} \simeq 10^{-19}-10^{-20}\right)$. The expected time delay after formation can be as low as about a few ten of thousand years.

Williams et al. $(2014,2016)$ report the results of a survey of M31 novae in quiescence. The derived catalog contains data for 38 spectroscopically confirmed novae from 2006 to 2012. They used Liverpool Telescope images of each nova during eruption to define an accurate position for each system. These positions were then matched to archival Hubble Space Telescope (HST) images and they performed photometry on any resolved objects that were coincident with the eruption positions. This in order to facilitate a search for their progenitor systems within archival Hubble Space Telescope (HST) data, with the aim of detecting systems with red giant secondaries (RG-novae) or luminous accretion disks. They found an elevated proportion of nova systems with evolved secondaries that may imply the presence of a much larger population of recurrent novae than previously thought. This would have considerable impact, particularly with regards to their potential as TypeIa SN progenitors. Their results also imply that RG-novae in M31 are more likely to be associated with the M31 disk population than the bulge, indeed the results are consistent with all RG-novae residing in the disk. If this result is confirmed in other galaxies, it suggests any Type-Ia SNe that originate from RG-nova systems are more likely to be associated with younger populations, and may be rare in old stellar populations, such as early-type galaxies.

An important paper by Churazov et al. (2014) reports the first ever detection of ${ }^{56} \mathrm{Co}$ lines at 847 and $1237 \mathrm{keV}$ and a continuum in the $200-400 \mathrm{keV}$ band from the Type-Ia SN2014J in M82 with INTEGRAL observatory. The data were taken between 50th and 100th day since the SN2014J outburst. The line fluxes suggest that $0.62 \pm 0.13 \mathrm{M}_{\odot}$ of radioactive ${ }^{56} \mathrm{Ni}$ were synthesized during the explosion. Line broadening gives a characteristic ejecta expansion velocity $\mathrm{V}_{\mathrm{e}} \sim 2100 \pm 500$ $\mathrm{km} \mathrm{s}^{-1}$. The flux at lower energies (200-400 keV) is consistent with the three-photon positronium annihilation, Compton downscattering and absorption in the $\sim 1.4 \mathrm{M}_{\odot}$ ejecta composed from equal fractions of iron-group and intermediate-mass elements and a kinetic energy $\mathrm{E}_{\mathrm{k}} \sim 1.4 \times 10^{51}$ erg. All these parameters are in broad agreement with a "canonical" model of an explosion of a Chandrasekhar-mass WD, providing an unambiguous proof of the nature of Type-Ia SNe as a thermonuclear explosion of a solar mass compact object. Late optical spectra (day 136 after the explosion) show rather symmetric $\mathrm{Co}$ and $\mathrm{Fe}$ line profiles, suggesting that, unless the viewing angle is special, the distribution of radioactive elements is symmetric in the ejecta (Churazov et al., 2015).

An interesting review about Progenitors of Core-Collapse Supernovae discussed on the pro- 
genitors of other types of SNe (Smartt, 2009) in order to clarify the high energetic explosions. The physical mechanism is still under debate, and these SNe pose a challenge to stellar evolution theory.

In my opinion, it is mandatory to mention the excellent review paper by Parrent, Friesen \& Parthasarathy (2014) about the type Ia supernova spectra. Type Ia supernovae have been primarily studied insofar as parameters discretized by relative epochs and incomplete temporal snapshots near maximum light. The authors discussed a necessary next step toward consistently modeling and directly measuring spectroscopic observables of type Ia supernova spectra. In addition, they analyzed current spectroscopic data in the parameter space defined by empirical metrics, which will be relevant even after progenitors are observed and detailed models are refined. A detailed analysis of the SN spectra can be useful also for determining the probable progenitors.

Maeda \& Terada (2016) discussed about the knowledge of the problem connected with the natures of progenitors of SNe Ia concluding that it is not yet clarified. There has been long and intensive discussion on whether the so-called SD scenario or the DD scenario, or anything else, could explain a major population of $\mathrm{SNe} \mathrm{Ia}$, but the conclusion has not yet been reached. With rapidly increasing observational data and new theoretical ideas, the field of studying the SN Ia progenitors has been quickly developing, and various new insights have been obtained in recent years. In their article they provide a summary of the current situation regarding the SN Ia progenitors, both in theory and observations. It seems difficult to explain the emerging diversity seen in observations of SNe Ia by a single population, and we emphasize that it is important to clarify links between different progenitor scenarios and different sub-classes of SNe Ia. A help for this purpose can be found in the review paper by Parrent, Friesen \& Parthasarathy (2014).

Benetti et al. (2005) studied the observational properties of 26 well-observed SN Ia with the intent of exploring SN Ia diversity. Based on the observed projected Doppler velocity evolution from the spectra, in conjunction with characteristics of the light curve $\left(\mathrm{M}_{\mathrm{B}}, \Delta \mathrm{m}_{15}\right)$, they considered three different groups of SN Ia: (1) "FAINT" SN 1991bg-likes, (2) "low velocity gradient" (LVG) SN 1991T/1994D-likes, and (3) "high velocity gradient" (HVG) SN 1984A-like events. The velocity gradient here is based on the time-evolution of 6100 ("6150") $\AA$ absorption minima as inferred from Si II $\lambda 6355$ line velocities. Overall, HVG SN Ia have higher mean expansion velocities than FAINT and LVG SN Ia, while LVG SN Ia are brighter than FAINT and HVG SN Ia on average (Silverman, Kong \& Filippenko, 2012; Blondin et al. 2012). Given an apparent separation of SN Ia subgroups from this sample of 26 objects, Benetti et al. (2005) considered it as evidence that LVG, HVG, and FAINT classifications signify three distinct kinds of SN Ia. However, these "three classes" are smoothly connected each other like shown in Fig. 17 (Parrent, Friesen \& Parthasarathy, 2014), as derived by the cluster analysis of Benetti (2005). This is one example more about the continuity among all the components of the Universe that I claimed many times.

Many attempts have been performed in order to find correlations among the different type-Ia $\mathrm{SNe}$ and their progenitors. The difficulty is connected to the lack of clear experimental indicators that can be used for the validation of models. The theoretical Chinese school is very active in this field. In the last few years they produced an interesting set of papers, as described below.

SNe Ia are believed to be thermonuclear explosions of carbon-oxygen white dwarfs (CO WDs). However, the mass accretion process onto CO WDs is still not completely understood. Wang et al. (2015) studied the accretion of He-rich matter onto CO WDs and explore a scenario in which a strong wind forms on the surface of the WD if the total luminosity exceeds the Eddington limit. 


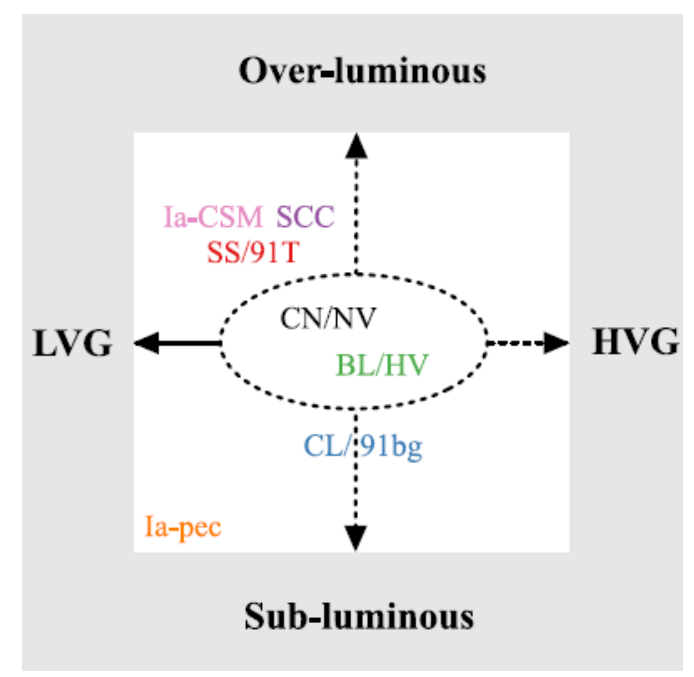

Figure 17: Subtype reference diagram. Dashed lines denote an open transitional boundary between adjacent spectroscopic subtypes (adopted from Parrent, Friesen \& Parthasarathy, 2014).

By using a stellar evolution code called modules for experiments in stellar astrophysics (MESA), they simulated the He accretion process onto CO WDs for WDs with masses of 0.6-1.35 $\mathrm{M}_{\odot}$ and various accretion rates of $10^{-8}-10^{-5} \mathrm{M}_{\odot} \mathrm{yr}^{-1}$. If the contribution of the total luminosity is included when determining the Eddington accretion rate, then a super-Eddington wind could be triggered at relatively lower accretion rates than those of previous studies based on steady-state models. The super-Eddington wind can prevent the WDs with high accretion rates from evolving into red-giant-like He stars. They found that the contributions from thermal energy of the WD are non-negligible, judging by their simulations, even though the nuclear burning energy is the dominating source of luminosity. They also provided the limits of the steady He-burning regime in which the WDs do not lose any accreted matter and increase their mass steadily, and calculated the mass retention efficiency during He layer flashes for various WD masses and accretion rates. These obtained results can be used in future binary population synthesis computations.

Recent observations suggest that some SNe Ia originate from the merging of two carbonoxygen white dwarfs (CO WDs). Meanwhile, hydrodynamical simulations have indicated that the accretion-induced collapse may be avoided under certain conditions when double WDs merge violently. However, the properties of SNe Ia from this violent merger scenario are highly dependent on a particular mass-accretion stage, the so-called WD + He subgiant channel, during which the primary WD is able to increase its mass by accreting He-rich material from an He subgiant before the system evolves into a double WD system. Liu et al. (2016) studied this particular evolutionary stage systematically and gave the properties of violent WD mergers. By employing the Eggleton stellar evolution code, they followed a large number of binary calculations and obtained the regions in parameter space for producing violent mergers based on the WD + He subgiant channel. According to these simulations, they found that the primary WDs can increase their mass by $\sim$ $0.10-0.45 \mathrm{M}_{\odot}$ during the mass-accretion stage. They then conducted a series of binary population synthesis calculations and found that the Galactic SN Ia birthrate from this channel is about 
$0.01-0.4 \times 10^{-3} \mathrm{yr}^{-1}$. This suggests that the violent WD mergers from this channel may only contribute to $\sim 0.3-10 \%$ of all SNe Ia in our Galaxy. The delay times of violent WD mergers from this channel are $\geq 1.7 \mathrm{Gyr}$, contributing to the SNe Ia in old populations. They also found that the $\mathrm{WD}+\mathrm{He}$ subgiant channel is the dominant way for producing violent WD mergers that may be able to eventually explode as SNe Ia.

The core-degenerate (CD) scenario has been proposed as an alternative way for the production of SNe Ia. Under this scenario, Wang et al. (2017) performed a detailed investigation based on a binary population synthesis approach. The SN Ia delay times from this scenario are basically in the range of 90-2500 Myr, mainly contributing to the observed SNe Ia with short and intermediate delay times, although this scenario can also produce some old SNe Ia. Their work indicates that the Galactic birthrates of SNe Ia from this scenario are not more than $20 \%$ of total SNe Ia due to more careful treatment of mass transfer. Although the SN Ia birthrates (in this work) are lower than those in Ilkov \& Soker $(2012,2013)$, the CD scenario cannot be ruled out as a viable mechanism for the formation of SNe Ia. Especially, SNe Ia with circumstellar material from this scenario contribute to 0.7-10\% of total SNe Ia, which means that the CD scenario can reproduce the observed birthrates of SNe Ia like PTF 11kx. They also found that SNe Ia happen systemically earlier for a high value of metallicity and their birthrates increase with metallicity.

Recently Liu, Wang \& Han (2018) performed a series of binary population synthesis simulations for the DD model, in which the WD+He subgiant channel is calculated by interpolations in the SN Ia production grid. According to their standard model, the Galactic birth rate of SNe Ia is about $2.4 \times 10^{-3} \mathrm{yr}^{-1}$ for the WD+He subgiant channel of the DD model; the total birth rate is about $3.7 \times 10^{-3} \mathrm{yr}^{-1}$ for all channels, reproducing that of observations. Previous theoretical models still have deficit with the observed SNe Ia with delay times $<1$ Gyr and $>8$ Gyr. After considering the WD+He subgiant channel, they found that the delay time distributions are comparable with the observed results. Additionally, some recent studies proposed that the violent WD mergers are more likely to produce SNe Ia based on the DD model. They estimated that the violent mergers through the DD model may contribute to at most $16 \%$ of all SNe Ia.

Recent tentative of searching channels for SN Ia progenitors have been performed by Liu et al. (2019): they searched for the symbiotic channel. In order to systematically investigate the parameter space, Galactic rates, and delay time distributions of SNe Ia arising from the symbiotic channel they used a revised mass-transfer prescription. They found that this channel could produce $\mathrm{SNe}$ Ia with intermediate and old ages, contributing up to $5 \%$ of all SNe Ia in the Galaxy. Their model increases the SN Ia rate from this channel by a factor of five. They suggest that the symbiotic systems RS Oph and T CrB are strong candidates for the progenitors of SNe Ia.

For comments and prospects about Type-Ia SN science in the decade 2010-2020 see the paper by Howell et al. (2009).

\section{Nuclear Reactions in Stars}

As I noted in Section 4, Starrfield, Iliadis \& Hix (2016) reported on the effects of improving both the nuclear reaction rate library and including a modern nuclear reaction network in their onedimensional, fully implicit, hydrodynamic computer code for describing both the recent advances 
in our understanding of the progress of the outburst and outline some of the puzzles that are still outstanding in the study of the thermonuclear runaway and the $\mathrm{CNe}$ outbursts.

For this reason it seems appropriate to me to spend a few words on the nuclear reactions in the stars.

It is difficult to date the beginning of the history of the study of the nuclear reactions in stars. However, Salpeter (1952a,b), Hoyle (1954), Cameron (1955), and Hoyle \& Schwarzschild (1955) indicated that the stable elements will be synthesized from hydrogen in the interior of evolving stars. Fowler, Burbidge, G.R. \& Burbidge, E.M. (1955) in their important paper on Stellar Evolution and the Synthesis of the Elements discussed the problem of transformation of hydrogen into helium by the pp-chain and CN-cycle. Fowler \& Greenstein (1956) discussed on the ElementBuilding Reactions in Stars, mainly with the synthesis of the $\sim 2 \%$ by mass of the elements heavier than $\mathrm{He}$, which they loosely call "metals".

By using the table of the relative abundances of the elements compiled by Suess \& Urey (1956), and the relative plot, Frank-Kamenetskii (1959) deeply discussed on The Origin of the Chemical Elements remarking on the possible pathways of the synthesis of the elements from the viewpoint of nuclear physics, nuclear reactions, physical conditions in the interiors of stars, the theories of the pre-stellar formation of the elements, the test of the theory of neutron capture, heterogeneous stars, Helium reactions, sources of neutrons in stars, slow and rapid processes of neutron capture, nuclei with maximum neutron excess, the thermonuclear theory of the formation of the elements, scandium: the cosmochemical thermometer, the iron maximum, the bypassed nuclei, the formation of elements in processes of athermal acceleration, the $(\mathrm{p}, \mathrm{n})$ and $(\mathrm{p}, 2 \mathrm{n})$ reactions.

The exhaustive review on Synthesis of the Elements in Stars by Burbidge, E.M. et al. (1957) can be deemed as the "Bible". All the reactions discussed are strongly dependent on the cross sections.

Therefore, the knowledge of the cross-sections of nuclear reactions occurring in the stars appears as one of the most crucial points of all astroparticle physics. Direct measurements of the cross sections of the ${ }^{3} \mathrm{He}\left({ }^{4} \mathrm{He}, \gamma\right){ }^{7} \mathrm{Be}$ and ${ }^{7} \mathrm{Be}(\mathrm{p}, \gamma){ }^{8} \mathrm{Be}$ reactions of the $p p$ chain and ${ }^{14} \mathrm{~N}(\mathrm{p}, \gamma){ }^{15} \mathrm{O}$ reaction of the CNO-cycle will allow a substantial improvement in our knowledge on stellar evolution.

Wolschin (2003) published a very interesting paper about the history of the "Thermonuclear Processes in Stars and Stellar Neutrinos".

An impressive review about nuclear reactions (the pp chain and CNO cycles) has been published by Adelberger et al. (2011). They summarize and critically evaluate the available data on nuclear fusion cross sections important to energy generation in the Sun and other hydrogen-burning stars and to solar neutrino production. Recommended values and uncertainties are provided for key cross sections, and a recommended spectrum is given for ${ }^{8} \mathrm{~B}$ solar neutrinos. They also discuss opportunities for further increasing the precision of key rates, including new facilities, new experimental techniques, and improvements in theory. This review, which summarizes the conclusions of a workshop held at the Institute for Nuclear Theory, Seattle, in January 2009, is intended as a 10-year update and supplement to the reviews by Adelberger et al. (1998).

It is in the nature of astrophysics that many of the processes and objects one tries to understand are physically inaccessible. Thus, it is important that those aspects that can be studied in the laboratory be rather well understood. 
One such aspect are the nuclear fusion reactions, which are at the heart of nuclear astrophysics: they influence sensitively the nucleosynthesis of the elements in the earliest stages of the universe and in all the objects formed thereafter, and control the associated energy generation, neutrino luminosity, and evolution of stars.

At the moment the LUNA (Laboratory for Underground Nuclear Astrophysics) is a new experimental approach for the study of nuclear fusion reactions based on an underground accelerator laboratory.

It is devoted to measure nuclear cross sections relevant in astroparticle physics. It is the most valuable experiment running underground in the Gran Sasso Laboratory of the INFN. Reviews about LUNA experiment have been published by Broggini et al., 2010, 2016a,b, 2017, 2018).

The LUNA experiment deals with reproducing in the laboratory the nuclear reactions that generate most of the energy produced by the stars and that have allowed the synthesis of the elements within the stars and in the early Universe. These reactions are characterized by a very small probability (cross section) to the energies of astrophysical interest and are very difficult to measure in laboratories on the earth's surface where the cosmic background would mask the weak expected signal. In the last 25 years the LUNA collaboration has installed two accelerators in the LNGS (Laboratori Nazionali del Gran Sasso - INFN) underground laboratories and measured some key reactions in the hydrogen combustion cycle and primordial nucleosynthesis. In the near future a new accelerator will be installed that will also enable reactions of helium and carbon combustion cycles to be measured (Prati et al., 2017; Cavanna et al., 2018; Gustavino et al., 2019).

The LUNA collaboration has already measured with good accuracy the key reactions $\mathrm{D}(\mathrm{p}, \gamma)^{3} \mathrm{He}$, ${ }^{3} \mathrm{He}(\mathrm{D}, \mathrm{p}){ }^{4} \mathrm{He}$ and ${ }^{3} \mathrm{He}\left({ }^{4} \mathrm{He}, \gamma\right){ }^{7} \mathrm{Be}$. These measurements substantially reduce the theoretical uncertainty of $\mathrm{D},{ }^{3} \mathrm{He},{ }^{7} \mathrm{Li}$ abundances. The $\mathrm{D}\left({ }^{4} \mathrm{He}, \gamma\right){ }^{6} \mathrm{Li}$ cross section - which is the key reaction for the determination of the primordial abundance of ${ }^{6} \mathrm{Li}$ - has been measured (e.g. Gustavino, 2007, 2009, 2011a,b, 2012, 2013), as well as that of ${ }^{2} \mathrm{H}(\alpha, \gamma){ }^{6} \mathrm{Li}$ (Anders et al., 2013), and ${ }^{2} \mathrm{H}(\alpha, \gamma){ }^{6} \mathrm{Li}$ (Anders et al., 2014).

Other reactions fundamental for a better knowledge of stellar evolution have been studied by the LUNA experiment: e.g. ${ }^{17} \mathrm{O}(\mathrm{p}, \gamma){ }^{18} \mathrm{~F}$ (Scott et al. 2012); ${ }^{25} \mathrm{Mg}(\mathrm{p}, \gamma){ }^{26} \mathrm{Al}$ (Strieder et al., 2012) ${ }^{25} \mathrm{Mg}(\mathrm{p}, \gamma){ }^{26} \mathrm{Al}$ (Straniero et al., 2013); ${ }^{17} \mathrm{O}(\mathrm{p}, \gamma){ }^{18} \mathrm{~F}$ (Di Leva et al., 2014).

Cavanna et al. (2015) studied with the LUNA experiment the ${ }^{22} \mathrm{Ne}(\mathrm{p}, \gamma)^{23} \mathrm{Na}$ reaction that takes part in the neon-sodium cycle of hydrogen burning. This cycle affects the synthesis of the elements between ${ }^{20} \mathrm{Ne}$ and ${ }^{27} \mathrm{Al}$ in asymptotic giant branch stars and novae. They found a new reaction rate a factor of 5 higher than the recent evaluation at temperatures relevant to novae and asymptotic giant branch stars nucleosynthesis.

Depalo et al. (2016) performed direct measurements of the ${ }^{22} \mathrm{Ne}(\mathrm{p}, \gamma)^{23} \mathrm{Na}$ resonances with the LUNA experiment. Based on the present experimental data and also previous literature data, an updated thermonuclear reaction rate is provided in tabular and parametric form. The new-reaction rate is significantly higher than previous evaluations at temperatures of 0.08-0.3 GK.

The ${ }^{17} \mathrm{O}(\mathrm{p}, \alpha){ }^{14} \mathrm{Na}$ reaction plays a key role in various astrophysical scenarios, from asymptotic giant branch stars to classical novae. It affects the synthesis of rare isotopes such as ${ }^{17} \mathrm{O}$ and ${ }^{18} \mathrm{~F}$, which can provide constraints on astrophysical models. Bruno et al. (2016) performed direct determination of the resonance strength $\mathrm{E}_{\mathrm{R}}=64.5 \mathrm{keV}$ of that reaction at the LUNA accelerator. They found a factor of 2 increase in the reaction rate at astrophysical temperatures relevant 
to shell hydrogen burning in red giant and asymptotic giant branch stars. The new rate implies lower ${ }^{17} \mathrm{O} /{ }^{16} \mathrm{O}$ ratios, with important implications on the interpretation of astrophysical observable quantities from these stars, as deeply discussed by Straniero et al. (2017).

In order to further remark the importance of LUNA measurement, we would like to mention the crucial problem of the correct prediction of the abundances of the light nuclides produced during the epoch of Big Bang Nucleosynthesis (BBN) which is one of the main topics of modern cosmology. Trezzi et al. (2017) report results about the cross section of the ${ }^{2} \mathrm{H}(\alpha, \gamma)^{6} \mathrm{Li}$ reaction that controls ${ }^{6} \mathrm{Li}$ production in the Big Bang. The cross section has been directly measured at the energies of interest for BBN for the first time, at center-of-mass energy $\mathrm{E}_{\mathrm{cm}}=80,93,120$, and 133 $\mathrm{keV}$. They found that the ${ }^{2} \mathrm{H}(\alpha, \gamma)^{6} \mathrm{Li}$ thermonuclear reaction rate is even lower than previously reported, thus increasing the discrepancy between predicted Big Bang ${ }^{6} \mathrm{Li}$ abundance and the amount of primordial ${ }^{6} \mathrm{Li}$ inferred from observations.

A general data base for Experimental Nuclear Reaction Data (EXFOR) can be found in: https://www-nds.iaea.org/exfor/exfor.htm.

\section{Some Open Questions}

Although dated it is important to remember the book Cataclysmic Variables and Related Objects edited by Margherita Hack and Costanze La Dous (1993) where all kind of cataclysmic objects are widely treated. The article by Hack (1993) on the continuous emission in high energy from CVs and related objects also deserves attention. It is mandatory to point out the excellent long review on Observations and Theory of Cataclysmic Variables: On Progress and Problems in Understanding Dwarf Novae and Nova-Like Stars by La Dous (1994) in which she deeply discuss on the problem of connecting theories versus experiments, and in spite of many good results, a lot of problems are still open.

These articles, in addition to discussing the behavior of cataclysmic systems, pose some open questions, many of which have not yet been fully resolved, despite almost 30 years having passed.

Indeed, several fundamental questions concerning CVs still remain waiting for a proper answer. Here I will present briefly only some of them.

One of them is the lack of a coherent classification, especially for NLs. On the other hand, in gross features and in most respects, DN and NLs, as well as quiescent novae, are almost indistinguishable, although, in addition to their different outbursts' behaviour, there appear to be some further minor differences which are not yet understood (see Hack et al., 1993). The question arises of whether the outburst behaviour, the current basis of almost all classification is really a suitable criterion for sorting CVs in physically related groups. There are also too many exceptions, either systems that do not fit in any particular group or that can be included in several of them, to be able to render the observational behaviour, at least as it is used at the present, suitable.

Could CVs be considered simply gravimagnetic rotators? This should be the most suitable approach for studying them from a physical point of view.

Studies of rotational equilibria of MCVs predict that IPCVs will evolve either into PCVs or into low field strength polars - presumably unobservable, and possibly EUV emitters - depending on their magnetic moments and orbital periods. Indeed, there are systems, like EX Hya-type, 
having magnetic moment similar to IPCVs above the 'period gap' and comparable to the weakest field AM Her-like systems.

Moreover, the detection of several SW Sex systems having orbital periods inside the so-called 'period gap' opens a new interesting problem about the continuity in the evolution of CVs.

The rare AM CVn stars have extremely short orbital periods, between 10 and 65 minutes, and their spectra show no evidence for hydrogen. They appear to be helium-rich versions of CVs. They are still waiting for a general model. They are probably binary systems of two white dwarfs, but even this is still controversial.

Despite all the work developed during the last decades, the problem of modeling accretion disks in CVs is by no means closed, especially in quiescence. Closely related is the problem of the cause of outbursts. We really do not know which of the present two families of models (Disk Instability Models or Secondary Instability Models) is responsible for the CVs outburst phenomenon, or in which system is each model valid, although Martinez-Pais et al. (1996) gave a contribution in solving this problem at least in the case of SS Cygni; they found some evidence for an increase of the mass transfer rate from the secondary star as the mechanism responsible for symmetric outbursts. Something similar can be said about the super-outburst phenomenon in SU UMa systems.

Gaudenzi et al. (1990), analyzing IUE spectra of SS Cygni, discussed about the outburst production as due to the destruction of the accretion disk. The matter slowly accretes onto the WD. Long and short outbursts correspond to total or partial destruction of the disk, respectively.

Alternatively, could nuclear burning be responsible of the production of outbursts in CVs? Indeed, nuclear burning onto white dwarf' surface was proposed by Mitrofanov $(1978,1980)$ as a mechanism suitable to generate X-rays in CVs. In spite of this shrewd suggestion, the community of theoreticians did not consider such a mechanism - certainly possible - worthy of taking up a part of their time. However, I believe that this alternative solution in explaining the generation of outbursts in CVs would deserve theoretician community's care. For instance, the white dwarf surface interested in the accretion in the system SS Cygni has been evaluated as $24 \%$ of the total (Gaudenzi et al., 2002). There, nuclear burning could occur.

Accretional heating by periodic DN events increases substantially the surface temperature of the WD in CVs (Godon \& Sion, 2002). Then, the envelope thermal structure resulting from compression and irradiation should be a crucial component in understanding the envelope structure of a pre-nova WD.

Another problem still open is connected with the classification of CVs in three kinds, namely NMCVs, PCVs and IPCVs. This is, in our opinion, another convenient classification, although artificial, probably not necessary if $\mathrm{CVs}$ are studied as gravimagnetic rotators. In this way a smooth evolution of the systems could be responsible of the variations of the gravimagnetic parameters.

Are the IPCVs and PCVs smoothly connected via the SW Sex-like systems placed just in between? SW Sex systems have indeed orbital periods belong to the so-called 'period gap', and then their presence there sure cancel that gap.

Could some systems behave in different ways depending on their instantaneous physical conditions? For this reason they could apparently behave sometimes as PCVs and sometimes as NPCVs.

An example very clear is that of SS Cygni, usually classified as a non-magnetic dwarf nova. It has been detected by the INTEGRAL observatory in a region of the spectrum (up to $\sim 100$ $\mathrm{keV}$ ). This emission is very hard to be explained without the presence of polar caps in the WD of 
the system. Several proofs have been shown and discussed many times by Giovannelli's group in order to demonstrate the Intermediate Polar nature of it (e.g., Giovannelli, 1996, and references therein; Giovannelli \& Sabau-Graziati, 1998; 2012a); indeed, SS Cygni shows characteristics of a NMCV, as well as those of IP and sometimes even those of polars, although its position in the $\log \mathrm{P}_{\text {spin }}-\log \mathrm{P}_{\text {orb }}$ plane is very close to the line where IPs lie.

Important results are coming from the SPITZER space telescope with the detection of an excess (3-8) $\mu \mathrm{m}$ emission from MCVs, due to dust (Howell et al., 2006; Brinkworth et al., 2007). Gaudenzi et al. (2011) discussed about the reasons of the variable reddening in SS Cyg and demonstrated that this reddening is formed by two components: the first is interstellar in origin, and the second (intrinsic to the system itself) is variable and changes during the evolution of a quiescent phase. Moreover, an orbital modulation also exists. The physical and chemical parameters of the system are consistent with the possibility of formation of fullerenes.

The SPITZER space telescope detected the presence of fullerenes in a young planetary nebula (Cami et al., 2010). Fullerenes are the first bricks for the emergence of the life. Therefore, the possible presence of fullerenes in CVs opens a new line of investigation, foreboding of new interesting surprises.

Further information can be considered in order to better synthesize the open problems in the knowledge of CVs and related objects.

Sion (http://astronomy.villanova.edu/faculty/sion/CV/index.html) states that in the Galaxy we could expect $\approx 10^{6} \mathrm{CVs}$. One of the big questions that arises is: "can all of the observed CVs and the phenomena associated with them be understood in terms of a single unified picture?" Other questions relate to the relative probabilities that $\mathrm{CVs}$ will be observed at particular stages in their evolution, and how the observations of $\mathrm{CVs}$ at the current epoch can be used to determine their ultimate fate. To address these questions Nelson (2012) and Goliasch \& Nelson (2015) have undertaken a massive computational effort to theoretically simulate the evolution of most of the possible $\mathrm{CVs}$ that could be produced by nature. The temporal evolution of 56,000 nascent CVs was followed over an age of 10 billion years using the MESA stellar evolution code. According to Nelson, "This is the most ambitious analysis of the properties of an entire CV population that has ever been undertaken. The whole project required several core-years of CPU time."

While many of the results confirmed what had already been inferred about the properties of $\mathrm{CVs}$, there were a number of surprises including the identification of a number of previously unexplored evolutionary pathways. But, as expected, a sharp bifurcation was found between nascent CVs that evolved to produce double white-dwarf binaries (including ones containing helium and hybrid white dwarfs), and ones that continuously transferred mass over the lifetime of the universe. In addition, the predictions of the theoretical simulations were in good general agreement with the observations of CVs with reasonably well-measured properties.

What was surprising was the large number of short-period "ultracompact" binaries (AM CVn stars) that were produced and, especially, the enormous depletion of carbon relative to nitrogen and oxygen that is predicted at certain epochs for evolved systems. As Nelson points out, "It seems that nature has provided us with a unique way to identify CVs that descended from a highly evolved state based on their carbon abundances. There is already some observational evidence to suggest that there is a significant depletion of carbon in certain CVs. This could be a really critical test that will allow us to infer the lineage of some CVs and predict what their fate will be". 
A paper by Otulakowska-Hypka, Olech \& Patterson (2016) present a statistical study of all measurable photometric features of a large sample of dwarf novae during their outbursts and superoutbursts. They used all accessible photometric data for all their objects to make the study as complete and up to date as possible. Their aim was to check correlations between these photometric features in order to constrain theoretical models which try to explain the nature of dwarf novae outbursts. They managed to confirm a few of the known correlations, that is the Stolz and Schoembs relation, the Bailey relation for long outbursts above the period gap, the relations between the cycle and supercycle lengths, amplitudes of normal and superoutbursts, amplitude and duration of superoutbursts, outburst duration and orbital period, outburst duration and mass ratio for short and normal outbursts, as well as the relation between the rise and decline rates of superoutbursts. However, they question the existence of the Kukarkin-Parenago relation but they found an analogous relation for superoutbursts. They also failed to find one presumed relation between outburst duration and mass ratio for superoutbursts. This study should help to direct theoretical work dedicated to dwarf novae.

Szkody \& Gänsicke (2012) provided a list of unanswered problems and questions and references for seeking additional information. Indeed, while the general evolutionary picture and the characteristics of the types of CVs are known at some level, there are major unsolved questions which remain. These include:

1. What is the actual number density and distribution of CVs in the Galaxy?

2. What happens to CVs once they reach the period minimum?

3. What are the detailed physics occurring in the common envelope?

4. What is the correct physics to describe viscosity in accretion disks?

5. What is the correct angular momentum prescription below the gap (besides gravitational radiation) that can account for the observed period minimum spike and the exact period distribution?

6. What causes the period gap?

7. How do Polars form and why are no magnetic white dwarfs in wide binaries observed? Are LARPS (Low Accretion Rate Polars) the progenitors of polars? Is there a difference in the emergence of systems containing magnetic white dwarfs versus non-magnetic?

8. What causes Polars, as well as the novalike disk systems with orbital periods between 3 and 4 hours, to cease mass transfer and enter low states? Are the associated mass transfer variations of the companion stars a general phenomenon among all CVs?

9. Can the white dwarfs in CVs grow in mass?

10. Do CVs contain exoplanets?

I can add one point more: 
11. Do CVs emit gravitational waves?

A possible answer to this point has been discussed by Poggiani (2017b), who reports that the most probable sources of GWs are AM CVn systems.

In order to answer to these not yet solved problems, a series of biennial Palermo Workshops about "The Golden Age of Cataclysmic Variables and Related Objects" has been organized since 2011, under my scientific direction. The refereed proceedings can be found in Giovannelli \& Sabau-Graziati (2012c; 2015b,c; 2017b).

\section{Conclusions}

At the end of this review it appears evident the role of the magnetic field intensity at the surface of the white dwarf in CVs. It was also remarked the importance of studying the evolutionary path of CVs that very probably is a continuous path connecting the so-called NMCVs with MCVs.

Indeed, the detection of several SW Sex systems having orbital periods inside the so-called 'period gap' opens a new interesting problem about the continuity in the evolution of CVs. Are the IPCVs and PCVs smoothly connected via the SW Sex-like systems placed just in between?

In order to fully understand the emission properties and evolution of CVs, the mass-transfer process needs to be clearly understood, especially magnetic mass transfer, as well as the properties of magnetic viscosity in the accretion disks around compact objects. Consequently, the investigation on the magnetic field intensities in WDs appears crucial in understanding the evolution of CVs systems, by which it is possible to generate classical novae (e.g., Isern et al., 1997) and type-Ia supernovae (e.g., Isern et al., 1993).

In those catastrophic processes the production of light and heavy elements, and then the knowledge of their abundances provides strong direct inputs for cosmological models and cosmic ray generation problems.

I want to conclude with a general warning, apparently underestimated, like discussed in Section 6: if we have not experimental information about the cross sections of nuclear reactions occurring in the stars it is hard to describe the correct star evolution.

The LUNA (Laboratory for Underground Nuclear Astrophysics) is devoted to measure nuclear cross sections relevant in astrophysics and astroparticle physics. It is the most valuable experiment running underground in the Gran Sasso Laboratory of the INFN. LUNA experiment provided the measures of the cross-sections of many nuclear reactions occurring in the stars for a better knowledge of stellar evolution.

As I discussed in the introduction, I can state that the Universe is interconnected in all its components: from cosmic network, to clusters of galaxies, to galaxies, to stars, to planets, to living beings, up to the simple bacterium. Therefore even every manifestation of life on our planet is subject to interconnection with all the surrounding environment. I can affirm that the whole Universe is a vital whole interconnected with more or less strong links between the various components, but that certainly exist.

Finally I can conclude with Fig. 18 that clearly explain all the mysteries of our Universe (Giovannelli, 2000), or if you prefer the same attempt written in Fig. 19. People who are able to 
read these sentences can understand that "The truth is written in the book of the Nature. We must learn to read this book".

$$
\begin{aligned}
& \text { 自然以山う教科書には } \\
& \text { 真果がある。 } \\
& \text { ただ我々は } \\
& \text { 読子方を学ば } \\
& \text { なければならない }
\end{aligned}
$$

Figure 18: Understanding our Universe (Giovannelli, 2000).

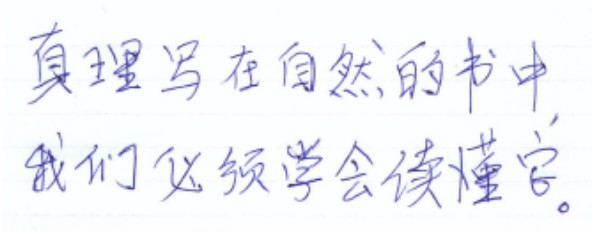

Figure 19: Understanding our Universe.

The experiments provide the basic alphabet, immersed in an apparently chaotic soup, but necessary to understand the nature. From that soup we must extract words and phrases to compose the book of the nature. In other words, the data coming from the experiments constitute the basic alphabet that we use for constructing models that attempt to describe the nature. But we have a lot of models for interpreting the experimental data by the light of science. Depending on the hypotheses the results could run against the experiments. Then, in order to be acceptable, models can take into account and justify $A \mathbf{L}$ the available data.

The same concept was expressed in much more incisive terms by Richard Phillips Feynman - Nobel laureate in Physics in 1965 - also known as The Great Explainer: It doesn't matter how beautiful your theory is, it doesn't matter how smart you are. If it doesn't agree with experiment, it's wrong.

\section{Acknowledgments}

This research has made use of "The NASA's Astrophysics Data System".

\section{References}

[1] Adelberger, E.G., Austin, S.M., Bahcall, J.N., Balantekin, A.B., Bogaert, G. et al.: 1998, Rev. Mod. Phys., 70, 1265-1291.

[2] Adelberger, E.G., García, A., Robertson, R.G. Hamish, Snover, K.A., Balantekin, A.B. et al.: 2011, Rev. Mod. Phys. 83, Issue 1, 195-246. 
[3] Aldering, G.: 2005, NewAR 49, 346-353.

[4] Anders, M., Trezzi, D., Bellini, A., Aliotta, M., Bemmerer, D. et al.: 2013, EPJA, 49, id. 28.

[5] Anders, M., Trezzi, D., Menegazzo, R., Aliotta, M., Bellini, A. et al. (LUNA Collaboration): 2014, Phys. Rev. Lett. 113, Issue 4, id. 042501.

[6] Bath, G.T., van Paradijs, J.: 1983, Nature, 305, 33.

[7] Becker, W., Trümper, J.: 1997, A\&A 326, 682-691.

[8] Benetti, S.: 2005, in 1604-2004: Supernovae as Cosmological Lighthouses, M. Turatto, S. Benetti, L. Zampieri \& W. Shea (Eds.), ASP Conf. Ser. 342, 235-241.

[9] Benetti, S., Cappellaro, E., Mazzali, P.A., Turatto, M., Altavilla, G.: 2005, ApJ. 623, Issue 2, 1011-1016.

[10] Bianchini, A.: 1990, in Physics of Classical Novae, A. Cassatella \& R. Viotti (eds.), Springer-Verlag, Lecture Notes in Physics, 369, 13.

[11] Bianco, F.B., Howell, D.A., Sullivan, M., Conley, A., Kasen, D., et al.: 2011, ApJ, 741, Issue 1, article id. 20, 12 pp.

[12] Bisikalo, D.V., Kononov, D.A., Kaigorodov, P.V., Zhilkin, A.G., Boyarchuk, A.A.: 2008, ARep 52, 318-326.

[13] Bisikalo, D.V., Zhilkin, A.G.: 2012, in From Interacting Binaries to Exoplanets: Essential Modeling Tools, M.T. Richards \& I. Hubeny (Eds.), IAU Symp. 282, 509-516.

[14] Bisikalo, D.V., Kurbatov, E.P., Pavlyuchenkov, Ya.N., Zhilkin, A.G., Kaygorodov, P.V.: 2016, MNRAS 458, 3892-3903.

[15] Blondin, S., Matheson, T., Kirshner, R.P., Mandel, K.S., Berlind, P.: 2012, AJ 143, Issue 5, article id. $126,33 \mathrm{pp}$.

[16] Bode, M.F.: 2010, Astron. Nachr. / AN331, No. 2, 160-168.

[17] Bode, M.F.: 2013, in The $11^{\text {th }}$ Asian-Pacific Regional IAU Meeting 2011, S. Komonjinda, Y. Kovalev \& D. Ruffolo (eds.), NARIT Conf. Ser., Vol. 1, 135-143.

[18] Bode, M.F., Evans, A. (eds.): 2008, Classical Novae, Cambridge Astrophys. Ser., No. 43, Cambridge University Press.

[19] Boneva, D., Kaigorodov, P.V., Bisikalo, D.V., Kononov, D.A.: 2009, ARep 53, 1004-1012.

[20] Boshkayev, K.: 2013, PhD Thesis, Sapienza University of Roma (Italy), online at https://pdfs.semanticscholar.org/2ead/5412338e1c93763248122726c9e9d1f99c7a.pdf

[21] Boshkayev, K., Rueda, J.A., Ruffini, R., Siutsou, I.: 2013, ApJ 762, Issue 2, article id. 117, 14 pp.

[22] Boshkayev, K., Rueda, J.A., Ruffini, R.: 2015, in The Thirteenth Marcel Grossmann Meeting: On Recent Developments in Theoretical and Experimental General Relativity, Astrophysics and Relativistic Field Theories, Proceedings of the MG13 Meeting on General Relativity (in 3 Volumes). Edited by ROSQUIST KJELL ET AL. Published by World Scientific Publishing Co. Pte. Ltd., pp. 2295-2300.

[23] Brinkworth, C.S., Hoard, D.W., Wachter, S., Howell, S.B., Ciardi, D.R., et al: 2007, in 15th European Workshop on WDs, R. Napiwotzki \& M. R. Burleigh (eds.), ASP Conf. Ser. 372, 333-336.

[24] Broggini, C.: 2016a, Journal of Physics: Conf. Ser. 703, Issue 1, article id. 012006. 
[25] Broggini, C.: 2016b, in Frontier Research in Astrophysics II, online at https://pos.sissa.it/cgi-bin/reader/conf.cgi?confid=269, id.5

[26] Broggini, C.: 2017, in 6th Roma International Conference on Astroparticle Physics, Morselli, A., Capone, A., Rodriguez Fernandez, G. (Eds.), EPJ Web of Conferences, Volume 136, id.01008.

[27] Broggini, C., Bemmerer, D., Guglielmetti, A., Menegazzo, R.: 2010, Annu. Rev. Nucl. Part. Sci. 60, 53-73.

[28] Broggini, C., Bemmerer, D., Caciolli, A., Trezzi, D.: 2018, Progr. in Part. and Nucl. Phys. 98, 55-84.

[29] Brown, W.R., Kilic, M., Allende Prieto, C.A., Kenyon, S.J.: 2011, MNRAS, 411, Issue 1, L31-L35.

[30] Bruno, C.G., Scott, D.A., Aliotta, M., Formicola, A., Best, A. et al. (LUNA Collaboration): 2016, Phys. Rev. Lett. 117, Issue 14, id.142502.

[31] Buckley, D.A.H., Meintjes, P.J., Potter, S.B., Marsh, T.R., Gänsicke, B.T.: 2017, Nat. As. 1, id. 0029.

[32] Burbidge, E.M., Burbidge, G.R., Fowler, W.A., Hoyle, F.: 1957, Rev. Mod. Phys. 29, Issue 4, 547-650.

[33] Cameron, A.G.W.: 1955, ApJ 121, 144-161.

[34] Cami, J., Bernard-Salas, J., Peeters, E., Malek, S.E.: 2010, Sci., 329, Issue 5996, 1180-1182.

[35] Camilo, F., Ransom, S.M., Halpern, J.P., Reynolds, J., Helfand, D.J. et al.: 2006, Nature, 442, Issue $7105,892-895$.

[36] Cassatella, A., Viotti, R. (eds.): 1990, Physics of Classical Novae, Springer-Verlag, Lecture Notes in Physics, 369.

[37] Cavanna, F., Depalo, R., Aliotta, M., Anders, M., Bemmerer, D. et al. (LUNA Collaboration): 2015, Phys. Rev. Lett. 115, Issue 25, id.252501.

[38] Cavanna, F. (on behalf of the LUNA collaboration): 2018, in International Symposium on t'Capture Gamma-Ray Spectroscopy and Related Topics (CGS16), Sun, Yang (Ed.), EPJ Web of Conferences, Volume 178, id.01007.

[39] Chiosi, E., Chiosi, C., Trevisan, P., Piovan, L., Orio, M.: 2015, MNRAS 448, Issue 3, 2100-2125.

[40] Christoforou, P.: 2017, at http://www.astronomytrek.com/10-interesting-facts-about-variable-stars

[41] Churazov, E., Sunyaev, R., Isern, J., Knödlseder, J., Jean, P. et al.: 2014, Nature 512, Issue 7515, 406-408

[42] Churazov, E., Sunyaev, R., Isern, J., Bikmaev, I., Bravo, E. et al.: 2015, ApJ 812, Issue 1, article id. $62,17 \mathrm{pp}$.

[43] Cooley, J.W., Tukey, J.W.: 1965, An algorithm for the machine calculation of complex Fourier series, Math. Comput. 19, 297-301.

[44] Cordova, F.A., Chester, T.J., Tuohy, I.R., Garmire, G.P.: 1980, ApJ 235, 163-176.

[45] Darnley, M.J., Ribeiro, V.A.R.M., Bode, M.F., Hounsell, R.A., Williams, R.P.: 2012, ApJ 746, Issue 1 , article id. $61,10 \mathrm{pp}$.

[46] Darnley, M.J., Bode, M.F., Harman, D.J., Hounsell, R.A., Munari, U. et al.: 2014, in Stella Novae: Past and Future Decades, P.A. Woudt \& V.A.R.M. Ribeiro (Eds.). ASP Conf. Ser. Vol. 490, 49-55.

[47] Depalo, R., Cavanna, F., Aliotta, M., Anders, M., Bemmerer, D. et al. (LUNA Collaboration): 2016, Phys. Rev. C. 94, 055804. 
[48] Di Leva, A., Scott, D.A., Caciolli, A., Formicola, A., Strieder, F. et al. (LUNA Collaboration): 2014, Phys. Rev. C, 89, Issue 1, id.015803 and erratum Phys. Rev C, 90, Issue 1, id.019902.

[49] Di Stefano, R.: 2010, ApJ 712, 728-733.

[50] Duncan, R.C., Thompson, C., 1992, ApJ 392, L9-L13.

[51] Enoto, T.: 2018, in Quarks and Compact Stars 2017 (QCS2017), JPS Conf. Proc., id.011046, 7 pp.

[52] Esposito, P., Rea, N., Israel, G.L.: 2018, arXiv:1803.05716.

[53] Evans, A.: 2011, in Asymmetric Planetary Nebulae 5 Conference, A.A. Zijlstra, F. Lykou, I. McDonald, \& E. Lagadec (eds.), Jodrell Bank Centre for Astrophysics, p. 305.

[54] Evans, A., Bode, M.F., O’Brien, T.J., Darnley, M.J. (eds.): 2008, RS Ophiuchi (2006) and the Recurrent Nova Phenomenon, ASP Conf. Ser., Vol. 401.

[55] Falck, B., Neyrinck, M.C.: 2015, MNRAS 450, Issue 3, 3239-3253.

[56] Ferrario, L., Wickramasinghe, D.T.: 2005, MNRAS 356, 615-620.

[57] Ferrario, L., Wickramasinghe, D.T.: 2007, ASP Conf. Ser. 372, 163-168.

[58] Ferrario, L., de Martino, D., Gänsicke, B.T.: 2015, SSRv 191, 111-169.

[59] Ferrario, L., Melatos, A., Zrake, J.: 2015, SSRv 191, 77-109.

[60] Fowler, W.A., Burbidge, G.R., Burbidge, E.M.: 1955, ApJ 122, 271-285.

[61] Fowler, W.A., Greenstein, J.L.: 1956, Proc. of the NAS 42, No 4, 173-180.

[62] Frank-Kamenetskii, D.A.: 1959, Soviet Physics Uspekhi 2, Issue 4, 600-619.

[63] Friedjung, M.: 1985, in Multifrequency Behaviour of Accreting Galactic Sources, F. Giovannelli (Ed.), SIDEREA, Roma, p. 370-371.

[64] Furlanetto, S.R., Oh, S.P.: 2016, MNRAS 457, 1813-1827.

[65] Gallagher, J.S., Starrfield, S.: 1978, ARA\&A 16, 171-214.

[66] Gänsicke, B.T.: 2005, in The Astrophysics of Cataclysmic Variables and Related Objects, J.-M. Hameury \& J.-P. Lasota (Eds.), ASP Conf. Ser. Vol. 330, 3.

[67] Gaudenzi, S., Giovannelli, F., Lombardi, R., Claudi, R.: 1990, AcA, 40, 105-115.

[68] Gaudenzi, S., Claudi, R.U., Giovannelli, F., Lombardi, R., Pelosi, M., Strappolini, M.: 2002, Mem. SAIt 73, 213-222.

[69] Gaudenzi S., Giovannelli, F., Mandalari, M., Corradini, M., Lombardi, R.: 2011, A\&A 525, id. A147, $11 \mathrm{pp}$.

[70] Giovannelli, F.: 1981, Space Sci. Rev. 30, Issue 1-4, 213-219.

[71] Giovannelli, F. (Ed.): 1985, Multifrequency of Accreting Galactic Sources, SIDEREA, Roma, 371 pp.

[72] Giovannelli, F.: 1985, in Multifrequency Behaviour of Galactic Accreting Sources, F. Giovannelli (Ed.), SIDEREA, Roma, p. 37.

[73] Giovannelli, F.: 1996, in Multifrequency Behaviour of High Energy Cosmic Sources, F. Giovannelli \& L. Sabau-Graziati (Eds.), Mem. S.A.It., 67, 401-413.

[74] Giovannelli, F.: 2000, in The Evolution of The Milky Way, F. Matteucci \& F. Giovannelli (Eds.), Kluwer Academic Publishers, pp. 619-620. 
[75] Giovannelli, F. (Ed.): 2001, The Bridge between the Big Bang and Biology (Stars, Planetary Systems, Atmospheres, Volcanoes: Their Link to Life), President Bureau of the CNR, Roma, Italy, pp. 1-440.

[76] Giovannelli, F.: 2017, in The Golden Age of Cataclismic Variables and Related Objects - IV, F. Giovannelli (Ed.), online at https://pos.sissa.it/cgi-bin/reader/conf.cgi?confid=315, id. 1 .

[77] Giovannelli, F., Gaudenzi, S., Rossi, C., Piccioni, A.: 1983, AcA 33, 319-330.

[78] Giovannelli, F., Martinez-Pais, I.G.: 1991, Space Sci. Rev. 56, 313-372.

[79] Giovannelli, F., Martinez-Pals, I.G., Sabau-Graziati, L.: 1992, in Viña del Mar Workshop on Cataclysmic Variable Stars, Nikolaus Vogt (Ed.), ASP Conf. Ser. 29, 119-124.

[80] Giovannelli, F., Sabau-Graziati, L.: 1998, in Ultraviolet Astrophysics Beyond the IUE Final Archive, W. Wamstecker \& R.Gonzalez-Riestra (eds.), ESA SP-413, 419-424.

[81] Giovannelli, F., Sabau-Graziati, L.: 1999, Mem. SAIt 70, 527-546.

[82] Giovannelli, F., Sabau-Graziati, L.: 2012a, in The Golden Age of Cataclysmic Variables and Related Objects, F. Giovannelli \& L. Sabau-Graziati (eds.), Mem. S.A.It., 83 N. 2, 698.

[83] Giovannelli, F., Sabau-Graziati, L.: 2012b, in Second Workshop on Robotic Autonomous Observatories, S. Guziy, S.B. Pandey, J.C. Tello \& A.J. Castro-Tirado (Eds.), Astronomical Society of India Conf. Ser. 7, 59-78.

[84] Giovannelli, F., Sabau-Graziati, L. (Eds.): 2012c, The Golden Age of Cataclysmic Variables and Related Objects - I, Mem. S.A.It., 83, N. 2, 433 pp.

[85] Giovannelli, F., Sabau Graziati, L.: 2015a, in The Golden Age of Cataclysmic Variables and Related Objects - III, Online at http://pos.sissa.it/cgi-bin/reader/conf.cgi?confid=255, id. 1.

[86] Giovannelli, F., Sabau Graziati, L. (Eds,): 2015b, The Golden Age of Cataclysmic Variables and Related Objects - II, Acta Polytechnica CTU proceedings, Vol. 2, 333 pp.

[87] Giovannelli, F., Sabau Graziati, L. (Eds,): 2015c, The Golden Age of Cataclysmic Variables and Related Objects - III, Online at http://pos.sissa.it/cgi-bin/reader/conf.cgi?confid=255.

[88] Giovannelli, F., Sabau-Graziati, L.: 2016a, in Accretion Processes in Cosmic Sources, online at http://pos.sissa.it/cgi-bin/reader/conf.cgi?confid=288, id. 1.

[89] Giovannelli, F., Sabau-Graziati, L.: 2017a, in Multifrequency Behaviour of High Energy Cosmic Sources - XII, Online at https://pos.sissa.it/cgi-bin/reader/conf.cgi?confid=306, id. 1.

[90] Giovannelli, F., Sabau Graziati, L. (Eds,): 2017b, The Golden Age of Cataclysmic Variables and Related Objects - IV, Online at https://pos.sissa.it/cgi-bin/reader/conf.cgi?confid=315.

[91] Giovannelli, F., Sabau-Graziati, L.: 2019, in Multifrequency Behaviour of High Energy Cosmic Sources - XIII, PoS-SISSA, online at https://pos.sissa.it/cgi-bin/chairman/chlist.cgi?confid=362, id. 003.

[92] Godon, P., Sion, E.M.: 2002, ApJ, 566, Issue 2, 1084-1090.

[93] Goliasch, J., Nelson, L.: 2015, ApJ 809, Issue 1, article id. 80, 19 pp.

[94] Greggio, L., Renzini, A., Daddi, E.: 2008, MNRAS 388, Issue 2, 829-837.

[95] Gustavino, C.: 2007, in Frontier Objects in Astrophysics and Particle Physics, F. Giovannelli \& G. Mannocchi (Eds.), Italian Physical Society, Ed. Compositori, Bologna, Italy, 93, 191-204. 
[96] Gustavino, C.: 2009, in Frontier Objects in Astrophysics and Particle Physics, F. Giovannelli \& G. Mannocchi (Eds.), Italian Physical Society, Ed. Compositori, Bologna, Italy, 98, 77-90.

[97] Gustavino, C.: 2011a, AIP Conf. Proc. 1377, 33-37.

[98] Gustavino, C.: 2011b, in Frontier Objects in Astrophysics and Particle Physics, F. Giovannelli \& G. Mannocchi (Eds.), Italian Physical Society, Ed. Compositori, Bologna, Italy, 103, 657.

[99] Gustavino, C.: 2012, in Nuclei in the Cosmos (NIC XII), http://pos.sissa.it/cgi-bin/reader/conf.cgi?confid=146, id.74

[100] Gustavino, C.: 2013, AcPol 53, 534-537.

[101] Gustavino, C. (on behalf of the LUNA collaboration): 2019, in RICAP18, 7th Roma International Conference on Astroparticle Physics, Edited De Vincenzi, M., Capone, A. \& Morselli, A. (Eds.), EPJ Web of Conferences, Volume 209, id.01043.

[102] Guy, J., Sullivan, M., Conley, A., Regnault, N., Astier, P., et al.: 2010, A\&A, 523, id. A7, 34 pp.

[103] Hack, M.: 1993, in Frontier Objects in Astrophysics and Particle Physics, F. Giovannelli \& G. Mannocchi (Eds.), Italian Physical Society, Ed. Compositori, Bologna, Italy, 40, 65-80.

[104] Hack, M., La Dous, C. (Eds.): 1993, Cataclysmic Variables and Related Objects, NASA SP-507.

[105] Hack, M., La Dous, C., Jordan, S.D., Stuart D., Thomas, R.N.: 1993, in Cataclysmic Variables and Related Objects, M. Hack \& C. la Dous (eds.), NASA SP-507, 1.

[106] Harding, A.K.: 2013, Front. Phys., 8, Issue 6, 679-692.

[107] Hartle, J.B., Thorne, K.S.: 1968, ApJ 153, 807-834.

[108] Hellier, C.: 2001, Cataclysmic Variable Stars, Springer.

[109] Hildebrand, R.H., Spillar, E.J., Stiening, R.F.: 1981, ApJ 243, 223-227.

[110] Hill, C.A., Smith, R.C., Hebb, L., Szkody, P.: 2017, MNRAS 472, 2937-2944.

[111] Horne, K.: 1985, MNRAS 213, 129-141.

[112] Horne, K., Gomer, R.: 1980, ApJ 237, 845-849.

[113] Howell, D.A., Conley, A., Della Valle, M., Nugent, P., Perlmutter, S., et al.: 2009, arXiv0903, $1086 \mathrm{H}$.

[114] Howell, S.B., Brinkworth, C., Hoard, D.W., Wachter, S., Harrison, T., et al.: 2006, ApJL 646, Issue 1, L65-L68.

[115] Hoyle, F.: 1954, ApJS 1, 121-146.

[116] Hoyle, F., Schwarzschild, M.: 1955, ApJS 2, 1-40.

[117] Hoyle, F., Fowler, W.A.: 1960, ApJ, 132, 565-590.

[118] Huang, S.-S.: 1972, ApJ 171, 549-564.

[119] Hudec, R.: 2015, in The Golden Age of Cataclysmic Variables and Related Objects - III, online at http://pos.sissa.it/cgi-bin/reader/conf.cgi?confid=255, id. 41.

[120] Hudec, R.: 2017, in Multifrequency Behaviour of High Energy Cosmic Sources - XII, online at https://pos.sissa.it/cgi-bin/reader/conf.cgi?confid=306, id. 83

[121] Hudec, R., Hudec, L., Klíma, M.: 2012, Acta Polytechnica, 52, No. 1, 27. 
[122] Hudec, R., Hudec, L.: 2013, Acta Polytechnica, 53, No. 3, 23.

[123] Iben, I., Jr., Tutukov, A.V.: 1984, ApJS, 54, 335-372.

[124] Ibrahim, A.I., Markwardt, C.B., Swank, J.H., Ransom, S., Roberts, M. et al.: 2004, ApJ 609, Issue 1, L21-L24.

[125] Ilkov, M,, Soker, N.: 2012, MNRAS 419, Issue 2, 1695-1700.

[126] Ilkov, M,, Soker, N.: 2013, MNRAS 428, Issue 1, 579-586.

[127] Isern, J., Hernanz, M., García-Berro, E.: 1993, in White Dwarfs: Advances in Observation and Theory, M.A. Barstow (ed.), Kluwer Academic Publ., Dordrecht, Holland, NATO ASI Ser., C403, 139.

[128] Isern, J., Hernanz, M., Abia, C., José, J.: 1997, in Frontier Objects in Astrophysics and Particle Physics, F. Giovannelli \& G. Mannocchi (eds.), Italian Physical Society, Editrice Compositori, Bologna, Italy, 57, 113.

[129] Joy, A.H.: 1956, ApJ 124, 317-320.

[130] Kaspi, V.M., Beloborodov, A.M.: 2017, ARA\$A 55, issue 1, 261-301.

[131] Kilic, M., Brown, W.R., Allende Prieto, C., Agüeros, M.A.; Heinke, C., Kenyon, S.J.: 2011, ApJ, 727, Issue 1 , article id. 3, 12 pp.

[132] Kiplinger, A.L.: 1979a, ApJ 234, 997-1015.

[133] Kiplinger, A.L.: 1979b, AJ 84, 655-660.

[134] Kistler, M.D., Stanek, K.Z., Kochanek, C.S., Prieto, J.L., Thompson, T.A.: 2013, ApJ, 770, Issue 2, article id. 88,8 pp.

[135] Kononov, D.A., Giovannelli, F., Bruni, I., Bisikalo, D.V.: 2012, A\&A 538, id. A94, 7 pp.

[136] Kotze, E.J., Potter, S.B., McBride, V.A.: 2015, A\&A 579, id. A77, 9 pp.

[137] Kotze, E.J., Potter, S.B., McBride, V.A.: 2016, A\&A 595, id. A47, 12 pp.

[138] Kowalski, M., Rubin, D., Aldering, G, Agostinho, R.J., Amadon, A., et al.: 2008, ApJ, 686, Issue 2, 749-778.

[139] La Dous, C.: 1994, Space Sci. Rev. 67, Issue 1-2, 1-221.

[140] Levitan, D.: 2013, Finding Needles in the Haystack: A Search for AM CVn Systems using the Palomar Transient Factory, Ph.D. Thesis, California Institute of Technology, U.S.A.

[141] Lipunov, V.M.: 1987, Ap\&SS 132, no. 1, 1-51.

[142] Lipunov, V.M.: 2018, talk at the Mondello Workshop 2018 in Frontier Research in Astrophysics - III.

[143] Lipunov, V.M., Postnov, K.A.: 1988, Ap\&SS 145, no. 1, 1-45.

[144] Lira, P.: 1995, Masters thesis, Univ. Chile.

[145] Liu, D.-D., Wang, B., Podsiadlowski, Ph., Han, Z.: 2016, MNRAS 461, Issue 4, 3653-3662.

[146] Liu, D., Wang, B., Han, Z.: 2018, MNRAS 473, Issue 4, 5352-5361.

[147] Liu, D., Wang, B., Ge, H., Chen, X., Han, Z.: 2019, A\&A 622, id. A35, 8 pp.

[148] Lombardi, R., Giovannelli, F., Gaudenzi, S.: 1987, Astroph. Space Sci. 130, Issue 1-2, 275-278.

[149] Luisi, P.L., Capra, F.: 2014, The systems View of Life, Cambridge Univ. Press, 510 pp. 
[150] Maeda, K., Terada, Y.: 2016, Int. J. Mod. Phys. D 25, Issue 10, id. 1630024.

[151] Maguire, K., Sullivan, M., Ellis, R.S., Nugent, P.E., Howell, D.A. et al.: 2012, MNRAS, 426, 2359.

[152] Malheiro, M., Rueda, J.A., Ruffini, R.: 2012, PASJ 64, No.3, article id. 56, 13 pp.

[153] Manchester, R.N.: 2017, Journal of Physics: Conf. Ser. 932, Issue 1, article id. 012002, 9 pp.

[154] Mannucci, F., Della Valle, M., Panagia, N.: 2006, MNRAS, 370, Issue 2, 773-783.

[155] Maoz, D., Mannucci, F.: 2012 PASA 29, Issue 4, 447-465.

[156] Maoz, D., Mannucci, F., Nelemans, G.: 2014, ARA\&A 52, 107-170.

[157] Marsh, T.R.: 2001, in Astrotomography, Indirect Imaging Methods in Observational Astronomy, H.M.J. Boffin, D. Steeghs \& J. Cuypers (Eds.), Lecture Notes in Physics 573, 1-27.

[158] Marsh, T.R., Horne, K.: 1988, MNRAS 235, 269-286.

[159] Marsh, T.R., Gänsicke, B.T., Hümmerich, S., Hambsch, F.-J., Bernhard, K., et al.: 2016, Nature 537, Issue $7620,374-377$.

[160] Martinez-Pais, I.G., Giovannelli, F., Rossi, C.; Gaudenzi, S.: 1994, A\&A 291, 455-467.

[161] Martinez-Pais, I.G., Giovannelli, F., Gaudenzi, S, Rossi, C.: 1996, A\&A, 308, 833-846.

[162] Mereghetti, S.: 2008, A\&A Rev. 15, 225-287.

[163] Mikołajewska, J.: 2013, in Binary Paths in Type Ia Supernovae Explosions, R. Di Stefano \& M. Orio (eds.), IAU Symp. 281, 162-165. (2011, arXiv: 1110.1847v1).

[164] Mitrofanov, I.G.: 1978, Sov. Astron. Lett., 4, 119-122.

[165] Mitrofanov, I.G.: 1980, in Close Binary Stars: Observations and Interpretations, D.M. Popper \& R.K. Ulrich (eds.), D. Reidel Publ. Co., Dordrecht, Holland, p. 431-436.

[166] Morini, M., Robba, N.R., Smith, A., van der Klis, M.: 1988, ApJ 333, 777-787.

[167] Nelson, L.: 2012, J. Phys.: Conf. Ser. Volume 341, Issue 1, id. 012008.

[168] Otulakowska-Hypka, M., Olech, A., Patterson, J.: 2016, MNRAS 460, Issue 3, 2526-2541.

[169] Paczynski, B.: 1990, ApJ 365, L9-L12.

[170] Pagnotta, A.S.: 2012, Recurrent novae and Type Ia supernova progenitors, Doctoral Dissertation, Louisiana State University (USA), LSU Digital Commons, 247 pp.

[171] Parkhurst, J.A., Zaccheus, D.: 1900, ApJ 12, 259-273.

[172] Parrent, J., Friesen, B., Parthasarathy, M.: 2014, Space Sci. Rev. 351, 1-52.

[173] Patat, F., Chandra, P., Chevalier, R., Justham, S., Podsiadlowski, Ph., et al.: 2008, The Messenger, 131, 30-34.

[174] Patterson, J.: 1979, Ph.D. Thesis, The University of Texas at Austin (USA).

[175] Patterson, J.: 1981, APJ Suppl Ser. 45, 517-539.

[176] Patterson, J.: 1992, ApJ 384, 234-248.

[177] Patterson, J., Robinson, E.L., Kiplinger, A.L.: 1978, ApJ 226, L137-L139.

[178] Patterson, J.: 2014, in 33rd Annual Symposium on Telescope Science, Society for Astron. Sci., 33, $17-22$. 
[179] Patterson, J., Uthas, H., Kemp, J., de Miguel, E., Krajci, Th.: 2013, MNRAS, 434, 1902.

[180] Pekurovsky, D.: 2012, Siam J. Sci. Comput. Vol. 34, No. 4, pp. C192ÜC209, Society for Industrial and Applied Mathematics.

[181] Phillips, M.M.: 2005, in 1604-2004: Supernovae as Cosmological Lighthouses, M. Turatto, S. Benetti, L. Zampieri, and W. Shea (Eds.), ASPC, 342, 211-216.

[182] Phillips, M.M.: 2012, Publ. Astron. Soc. Australia 29, Issue 4, 434-446. (2011, arXiv: 11.4463v1).

[183] Phillips, M.M., Lira, P., Hamuy, M., Maza, J.: 1999, AJ, 118, Issue 4, 1766-1776.

[184] Plavec, M.: 1968, Astroph. Space Sci. 1, Issue 2, 239-263.

[185] Podsiadlowski, Ph.: 1991, Nature 350, 684-685.

[186] Podsiadlowski, Ph.: 2017, in Handbook of Supernovae, A.W. Alsabti, P. Murdin (Eds.), Springer International Publishing AG, 1-14.

[187] Podsiadlowski, Ph., Morrisa, T.S., Ivanovac, N.: 2007, AIP Conf. Proc. 937, 125-133.

[188] Poggiani, R.: 2017a, in Multifrequency Behaviour of High Energy Cosmic Sources - XII, online at https://pos.sissa.it/cgi-bin/reader/conf.cgi?confid=306, id.53.

[189] Poggiani, R.: 2017b, in The Golden Age of Cataclysmic Variables and Related Objects - IV, in press.

[190] Poggiani, R.: 2019, in The Golden Age of Cataclysmis Variables and Related Objects - V, F. Giovannelli (Ed.), online at https://pos.sissa.it/cgi-bin/chairman/chlist.cgi?confid=368, id. 026.

[191] Pontzen, A., Governato, F.: 2014, https://www.flickr.com/photos/uclmaps/15051460475/.

[192] Prati, C. (on behalf of the LUNA collaboration): 2017, talk at the XV International Conference on Astroparticle and Underground Physics, 24-28 July, Subdury, ON, Canada.

[193] Rea, N., Esposito, P., Turolla, R., Israel, G.L., Zane, S et al.: 2010, Science 330, Issue 6006, 944-946.

[194] Rea, N., Israel, G.L., Esposito, P., Pons, J.A., Camero-Arranz, A. et al.: 2012, ApJ 754, Issue 1, article id. $27,13 \mathrm{pp}$.

[195] Rea, N., Israel, G.L., Pons, J.A., Turolla, R., Viganò, D. et al.: 2013, ApJ 770, Issue 1, article id. 65, $14 \mathrm{pp}$.

[196] Rea, N., Viganò, D., Israel, G.L., Pons, J.A., Torres, D.F.: 2014, ApJL 781, Issue 1, article id. L17, 4 pp.

[197] Rees, M.J.: 1988, in Origins, A.C. Fabian (ed.), Cambridge University Press, 1.

[198] Rodriguez-Gil, P.: 2003, Ph.D. Thesis, La Laguna University, Spain.

[199] Rueda, J.A., Boshkayev, K., Izzo, L., Ruffini, R., Lorén-Aguilar, P. et al.: 2013, ApJ 772, Issue 2, article id. L24, 4 pp.

[200] Salewski, M., Geiger, B., Heidbrink, W.W., Jacobsen, A.S., S B Korsholm, S.B., et al. (the ASDEX Upgrade Team): 2015, Plasma Phys. Control. Fusion 57, 014021, 10 pp.

[201] Salpeter, E.E.: 1952a, ApJ 115, 326-328.

[202] Salpeter, E.E.: 1952b, Phys. Rev. 88, Issue 3, 547-553.

[203] Sahni, V., Sathyaprakash, B.S., Shandarin, S.F.: 1997, ApJ 476, Issue 1, L1-L5. 
[204] Scalzo, R.A., Ruiter, A.J., Sim, S.A.: 2014, MNRAS, 445, Issue 3, 2535-2544.

[205] Scaringi, S., Bird, A.J., Norton, A.J., Knigge, C., Hill, A.B. et al.: 2010, MNRAS 401, Issue 4, 2207-2218.

[206] Schaefer, B.E.: 2010, ApJS, 187, 275.

[207] Schaefer, B.E.: 2011, ApJ, 742, 112.

[208] Schmidtobreick, L.: 2013, Central European Astrophysical Bulletin 37, 361-368.

[209] Schreiber, M.R., Lasota, J.-P.: 2007, A\&A 473, Issue 3, 897-901.

[210] Scott, D.A., Caciolli, A., Di Leva, A., Formicola, A., Aliotta, M. et al.: 2012, Phys. Rev. Lett, 109, Issue 20, id. 202501.

[211] Seitter, W.C..: 1990, in Physics of Classical Novae, A. Cassatella \& R. Viotti (eds.), Springer-Verlag, Lecture Notes in Physics, 369, 79.

[212] Shafter, A.W., Curtin, C., Pritchet, C.J., Bode, M.F., Darnley, M.J.: 2014, in Stella Novae: Past and Future Decades, P.A. Woudt \& V.A.R.M. Ribeiro (eds.), ASP Conf. Ser., 490, 77.

[213] Shafter, A.W., Henze, M., Rector, T.A., Schweizer, F., Hornoch, K. et al.: 2015, ApJS, 216, 34.

[214] Shandarin, S.F.: 1983, Sov. Astr. Lett. 9, Mar.-Apr., 104-106.

[215] Shandarin, S., Feldman, H.A., Heitmann, K., Habib, S.: 2006, MNRAS 367, Issue 4, 1629-1640.

[216] Shandarin, S., Habib, S., Heitmann, K.: 2010, PhRv D, 81, Issue 10, id. 103006.

[217] Silverman, J.M., Kong, J.J., Filippenko, A.V.: 2012, MNRAS 425, Issue 3, 1819-1888.

[218] Smak, J.: 1967, AcA, 17, 255-270.

[219] Smak, J.: 1969, AcA 119, 155-164.

[220] Smak, J.: 1981, AcA 31, 395-408.

[221] Smak, J.: 1985, in Multifrequency) Behaviour of Galactic Accreting Sources, F. Giovannelli (Ed.), SIDEREA, p. 3-16.

[222] Smartt, S.J.: 2009, ARA\&A 47, 63-106.

[223] Starrfield, S., Sparks, W.M.: 1987, Astroph. Space Sci. 131, 379-393.

[224] Starrfield, S., Iliadis, C., Hix, W.R.: 2016, PASP 128:051001, 17 pp.

[225] Stover, R.J., Robinson, E.L., Nather, R.E., Montemayor, T.J.: 1980, ApJ 240, 597-607.

[226] Straniero, O., Imbriani, G., Strieder, F., Bemmerer, D., Broggini, C. et al.: 2013, ApJ, 763, id. 100, $10 \mathrm{pp}$.

[227] Straniero, O., Bruno, C.G., Aliotta, M., Best, A., Boeltzig, A. et al.: 2017, A\&A 598, A128.

[228] Strieder, F., Limata, B., Formicola, A., Imbriani, G., Junker, M. et al.: 2012, PhL B, 707, Issue 1, 60-65.

[229] Stril, A., Cahn, R.N., Linder, E.V.: 2010, MNRAS 404, Issue 1, 239-246.

[230] Suess, H.E., Urey, H.C.: 1956, Revs. Modern Phys. 28, Issue 1, 53-74.

[231] Surina, F., Bode, M.F., Darnley, M.J: 2013, in The $11^{\text {th }}$ Asian-Pacific Regional IAU Meeting 2011, S. Komonjinda, Y. Kovalev \& D. Ruffolo (eds.), NARIT Conf. Ser., Vol. 1, 161-164. 
[232] Surina, F., Bode, M.F., Darnley, M.J: 2015, Publ. Korean Astr. Soc. 30, 237-240.

[233] Szkody, P., Gänsicke, B.T.: 2012, JAAVSO 40, no. 1, 563-571.

[234] Thompson, C., Duncan, R.C., 1995, MNRAS, 275, 255-300.

[235] Townsley, D.M., Bildsten, L.: 2005, ApJ, 628, Issue 1, 395-400.

[236] Trezzi, D., Anders, M., Aliotta, M., Bellini, A., Bemmerer, D. et al. (LUNA Collaboration): 2017, Astrop. Phys. 89, 57-65.

[237] Truran, J.W.: 2002, in The Physics of Cataclysmic Variables and Related Objects, B.T. Gänsicke, K. Beuermann \& K. Reinsch (Eds.), ASP Conf. Ser. 261, 576-584.

[238] Voss, R., Nelemans, G.: 2008, Nature, 451, Issue 7180, 802-804.

[239] Wang, B, Han, Z.: 2012, NewAR 56, Issue 4, 122-141.

[240] Wang, B., Liu, Z., Chen, H., Jia, S., Han, Z.: 2013, in Feeding Compact Objects: Accretion on All Scales, C.M. Zhang, T. Belloni, M. MMéndes \& S.N. Zhang (Eds.), IAU Symp. 290, 321-322.

[241] Wang, B., Li, Y., Ma, X., Liu, D.-D., Cui, X., Han, Z.: 2015, A\&A 584, id. A37, 6 pp.

[242] Wang, B., Zhou, W.-H., Zuo, Z.-Y., Li, Y.-B., Luo, X.: 2017, MNRAS 464, Issue 4, 3965-3971.

[243] Warner, B.: 2002, in Classical Nova Explosions, AIP Conf. Proc., 637, 3.

[244] Webbink, R.F.: 1984, ApJ, 277, 355-360.

[245] Whelan, J., Iben, I. Jr.: 1973, ApJ, 186, 1007-1014.

[246] Wickramasinghe, D.T., Tout, C.A., Ferrario, L.: 2014, MNRAS 437, 675-681.

[247] Williams, S.C., Darnley, M.J., Bode, M.F., Shafter, A.W.: 2014, in Stella Novae: Past and Future Decades, P.A. Woudt \& V.A.R.M. Ribeiro (eds.), ASP Conf. Ser., 490, 85-90.

[248] Williams, S.C., Darnley, M.J., Bode, M.F., Shafter, A.W.: 2016, ApJ. 817, Issue 2, article id. 143,14 pp.

[249] Wolschin, G.: 2003, in Time, Quantum and Information, Lutz Castell \& Otfried Ischebeck ((Eds.), Springer, Berlin, Germany, p. 115.

[250] Yaron, O., Prialnik, D., Shara, M.M., Kovetz, A.: 2005, ApJ, 623, 398.

[251] Yoon, S.-C., Podsiadlowski, Ph., Rosswog, S.: 2007, MNRAS, 380, Issue 3, 933-948.

[252] Zhilkin, A.G., Bisikalo, D.V.: 2009, in Numerical Modeling of Space Plasma Flows: ASTRONUM-2008, Nikolai V. Pogorelov, Edouard Audit, Phillip Colella \& Gary P. Zank (Eds.), ASP Conf. Ser. 406, 118-123.

[253] Zhilkin, A.G., Bisikalo, D.V.: 2011, in 5th international conference of numerical modeling of space plasma flows (astronum 2010), Nikolai V. Pogorelov, Edouard Audit \& Gary P. Zank, (Eds.), ASP Conf. Ser. 444, 91-96.

[254] Zhilkin, A.G., Bisikalo, D.V., Mason, P.A.: 2012, ARep 56, 257-274.

[255] Zuckerman, M.-C. 1961, Annales d'Astrophysique 24, 431. 